\title{
On-orbit calibration of geostationary electron and proton flux observations for augmentation of an existing empirical radiation model
}

\author{
Juan V. Rodriguez ${ }^{1,2, *}$, Michael H. Denton ${ }^{3,4}$, and Michael G. Henderson ${ }^{5}$ \\ ${ }^{1}$ Ball Aerospace, Boulder, 80301 CO, USA \\ 2 Formerly at CIRES, University of Colorado, Boulder, 80309 CO, USA \\ ${ }^{3}$ Space Science Institute, Boulder, $80301 \mathrm{CO}$, USA \\ ${ }^{4}$ New Mexico Consortium, Los Alamos, 87544 NM, USA \\ ${ }^{5}$ Los Alamos National Laboratory, Los Alamos, 87545 NM, USA
}

Received 11 February 2020 / Accepted 20 June 2020

\begin{abstract}
Challenges faced in the quantitative use of long-term radiation belt data sets include establishing their relative accuracy and correcting for on-orbit degradation. An existing empirical model of energetic plasmas (0.001-40 keV) in geosynchronous orbit has been extended in energy to several hundred keV by incorporating observations from the Geostationary Operational Environmental Satellite (GOES) 13 and 15 magnetospheric electron detector (MAGED) and magnetospheric proton detector (MAGPD). In order to ensure the accuracy of this energy range extension, the following steps were taken: (1) removing noise bursts; (2) intra-calibrating the nine solid state telescopes comprising each MAGED or MAGPD; (3) cross-calibrating GOES 13 and 15; and (4) cross-calibrating magnetospheric plasma analyzer (MPA) and GOES fluxes, the necessary final step in augmenting an MPA-based model with GOES data. The MAGED and MPA electron fluxes were demonstrated to agree well at the energy (40 keV) where they overlap, while the MAGPD proton fluxes exhibited a severe long-term degradation compared to MPA. This problem is related to the well-known long-term degradation of the proton fluxes from the similar medium energy proton and electron detector (MEPED) proton telescopes on the POES and Metop satellites in low-Earth orbit. Results of this on-orbit calibration work are used to reconsider long-standing hypotheses about the cause of degradation in similar proton telescopes.
\end{abstract}

Keywords: cross-calibration / on-orbit degradation / radiation damage / GOES / Los Alamos

\section{Introduction}

Empirical models of plasma and radiation populations in Earth's magnetosphere are valuable for a wide range of scientific and engineering purposes. When organized by physically relevant parameters such as local time, geomagnetic indices, and solar wind drivers, they provide a reliable baseline against which the results of observational studies or physics-based predictive models can be compared. Of interest are both the magnitude of short-term deviations from the inherently smoothed empirical models as well as systematic differences. Empirical models may also be used to characterize the range of harmful plasma and radiation environments that spacecraft must endure in the magnetosphere. A challenge faced in the development of empirical models that cover a wide energy range and a large volume of the magnetosphere is the combination of multiple

\footnotetext{
*Corresponding author: jvrod23@gmail.com
}

data sets from different instruments and different satellites. In order to achieve a self-consistent empirical model, this requires cross-calibration of the different data sets (e.g., Ginet et al., 2013).

Denton et al. (2015, 2016) have developed a geosynchronous ( $\sim 6.6$ Earth radii) plasma model from $>80$ satelliteyears of the Los Alamos magnetospheric plasma analyzer (MPA) electron and ion plasma measurements. This model describes $1 \mathrm{eV}-40 \mathrm{keV}$ electron and ion fluxes in geosynchronous orbit, organized into 24 magnetic local time bins and parameterized by the geomagnetic index $K p$ and the solar wind electric field $\left(-v_{\mathrm{sw}} \times B_{z}\right)$. The electron portion of this model has been extended radially outward in the equatorial plane to $\sim 6-20$ Earth radii at $45-325 \mathrm{keV}$ using data from the research with adaptive particle imaging detectors (RAPID) instrument on Cluster (Denton et al., 2019). Most recently, the upper end of the model energy range in geosynchronous orbit is being extended from $40 \mathrm{keV}-475 \mathrm{keV}$ for electrons 
and $575 \mathrm{keV}$ for protons using measurements from the Geostationary Operational Environmental Satellite (GOES)-13 and -15 magnetospheric electron and proton detectors (MAGE/PD).

Magnetospheric Electron Detector measures 30-600 keV electron fluxes, which represent a transition in magnetospheric dynamics from electric-field-dominated drift (Korth et al., 1999) at lower energies to gradient-curvature drift at higher energies (Sibeck et al., 1987). In the area of spacecraft effects, this energy range includes the transition regime from surface charging to internal charging effects, identified as $50-100 \mathrm{keV}$, with $80 \mathrm{keV}$ corresponding to a depth of 2 mils $(0.051 \mathrm{~mm})$ in aluminum (NASA, 2011). Zheng et al. (2019) have identified the $>10 \mathrm{keV}$ integral electron flux as being well-correlated with surface charging anomalies. Accurate calculation of this integral is one reason to extend the energy range of the Denton et al. $(2015,2016)$ model above $40 \mathrm{keV}$. (By their smoothed nature, such climatological models are not appropriate for identifying worst-case surface charging environments, for which see, for example, Matéo-Vélez et al., 2018).

Magnetospheric Proton Detector measures $80-800 \mathrm{keV}$ proton fluxes, which represent the higher portion of the energy range and the physical outer edge of the ring current (Zhao et al., 2015). Protons in this energy range are known to damage space systems. For example, in the late 1960's, several satellites in geosynchronous orbit suffered a rapid decrease in power output ( $20 \%$ within weeks of launch) that was determined to be due to exposure of incompletely-covered solar cells to low-energy proton fluxes (Hughes Aircraft Company, 1968; Statler \& Curtin, 1971; Tada et al., 1982). Subsequent laboratory testing with 150 and $270 \mathrm{keV}$ protons indicated that damage was possible if as little as $2 \%$ of the solar cell were uncovered (Statler \& Curtin, 1971). The phenomenon had not been observed prior to this since solar cells had been completely covered. Following the explanation of this phenomenon, the general practice has been to be completely cover semiconductor solar cells in space with thin coverglass to prevent lower energy particles from reaching the active material (Tada et al., 1982). Coverglass darkening by 10 's-100's of keV protons, particularly in the presence of contamination, a problem recognized on ATS-1 (Hughes Aircraft Company, 1968), continues to be an area of active research (e.g., Liu et al., 2011). On the Chandra X-ray Observatory, 100-300 keV protons damage the frontilluminated charge-coupled devices (CCDs) in the advanced CCD imaging spectrometer (ACIS), causing increased chargetransfer inefficiency and thus degraded energy resolution (O'Dell et al., 2000). Since 1999, this damage has been limited by moving the ACIS away from the observing position using the Science Instrument Module during radiation belt passes of the highly-elliptical Chandra orbit.

This paper describes the methods and results of the extensive on-orbit corrections and cross-calibrations applied to the GOES MAGED and MAGPD fluxes in order to make them suitable for augmenting the MPA-based empirical geosynchronous plasma sheet-radiation belt model of Denton et al. (2015, 2016). (By “on-orbit," we mean corrections and calibrations performed on the ground using data taken by multiple satellite instruments after launch, where the aim is to compute factors by which these data are made consistent). The MAGED electron fluxes have been found to agree well with the MPA data over 7+ years. In contrast, the MAGPD proton fluxes clearly show evidence of on-orbit degradation (and partial recovery) that have had to be corrected prior to incorporation into the model. Models of radiation damage used successfully to correct similar telescopes in lower-dose orbits are insufficient to explain the observed behavior of magnetospheric electron detector (MAGED) and magnetospheric proton detector (MAGPD) and therefore need augmentation for higher-dose orbits.

\section{Data}

The NOAA Space Weather Prediction Center (SWPC) started processing the GOES-13 and -15 MAGED and MAGPD fluxes in real time in late December 2010. Magnetic fields from both satellites are publicly available starting in January 2011. Therefore, for the augmentation of the empirical model, the fluxes have been corrected and converted into omnidirectional fluxes over the period January 2011 through December 2017 (for GOES-13) and December 2018 (for GOES-15). GOES13 was at $\sim 75^{\circ} \mathrm{W}$ during the entire period; GOES-15 drifted from $\sim 90^{\circ} \mathrm{W}$ to $\sim 135^{\circ} \mathrm{W}$ in October-December 2011. SWPC stopped receiving GOES-13 data on December 14, 2017. The GOES-15 MAGED and MAGPD instruments were turned off temporarily on December 17, 2018. Although the GOES-14 instruments were functional, their data were processed only sporadically before December 1,2017 , and therefore were not included in this effort. Particle fluxes from July 2010 used in the cross-calibration among the three satellites were processed from the raw data using algorithms similar to those used in the real-time ground processing. Fluxes are used in this study, rather than counts or count rates, because only fluxes are available in 1-min averages, and the pitch angles needed for calculation of omnidirectional fluxes are only available at 1-min cadence.

The GOES MAGED and MAGPD telescopes are briefly described here and compared to the POES/Metop space environment monitor (SEM)-2 medium energy proton and electron detector (MEPED) electron and proton telescopes, upon which their designs are based. This is followed by a brief description of the MPA.

A common feature of MAGED and MAGPD is the arrangement of their constituent telescopes such that their fields-of-view (FOVs) together form a cruciform field-of-regard composed of a vertical and horizontal fan. Each instrument comprises nine solid-state telescopes identical in design. The vertical (northsouth) fan is composed of five telescope FOVs with center angles at $0^{\circ}, \pm 35^{\circ}$ and $\pm 70^{\circ}$ from the equatorial plane, the $0^{\circ}$ telescope (Telescope 1 ) looking radially outward in the equatorial plane (toward zenith). The horizontal (east-west) fan is composed of four additional telescope FOVs with center angles at $\pm 35^{\circ}$ and $\pm 70^{\circ}$ from the north to south plane, as illustrated elsewhere (Rowland \& Weigel, 2012; Sillanpää et al., 2017). The MAGED and MAGPD channel names and energies are listed in Table 1. The MAGED and MAGPD energy response functions are documented, respectively, in Tables 4-10 and 5-9 of Hanser (2011).

The multiple telescopes provide adequate pitch-angle coverage on a three-axis-stabilized spacecraft. The pitch angles are calculated using the nominal orientation of each telescope and the components of the magnetic field in the spacecraft body 
Table 1. MAGED and MAGPD channel names and energies (Hanser, 2011).

\begin{tabular}{|c|c|c|c|c|c|}
\hline \multicolumn{3}{|c|}{ MAGED channels } & \multicolumn{3}{|c|}{ MAGPD channels } \\
\hline Name & Energy range $(\mathrm{keV})$ & $\overline{\text { Center energy }(\mathrm{keV})}$ & $\overline{\text { Name }}$ & Energy range $(\mathrm{keV})$ & $\overline{\text { Center energy }(\mathrm{keV})}$ \\
\hline ME1 & $30-50$ & 40 & MP1 & $80-110$ & 95 \\
\hline ME3 & $100-200$ & 150 & MP3 & $170-250$ & 210 \\
\hline ME4 & $200-350$ & 275 & MP4 & $250-350$ & 300 \\
\hline ME5 & $350-600$ & 475 & MP5 & $350-800$ & 575 \\
\hline
\end{tabular}

reference frame as measured by the GOES primary fluxgate magnetometer (Rodriguez, 2014). In this paper, the pitch angles are calculated at the telescope FOV centers from 1-min averages of the magnetic field components. An example of three days of GOES-13 pitch angles is shown in Figure 1. The typical quiettime diurnal variation of the pitch angles is evident on 4 July 2015 until $\sim 15$ UT and on 6 July 2015 after $\sim 9$ UT. The pitch angles between $\sim 18$ UT on 4 July and $\sim 6$ UT on 5 July are due to a highly stretched near-tail magnetic field, $K p$ being 5 or $5+$. This pitch angle variation during stretching illustrates how the number of cases of telescope pairs having the same pitch angle increases during disturbed periods. However, despite the disturbed conditions, telescope 9 has only one such pitch-angle match with another telescope during this period. Such cases, in which two telescopes observe fluxes at the same local pitch angle, form the basis for the telescope intra-calibration technique of Rowland \& Weigel (2012), applied in Section 4.2 to the MAGED and MAGPD data.

\subsection{MAGED}

Each MAGED telescope consists of a single $700-\mu \mathrm{m}$ surface-barrier silicon detector located behind two collimators and a nickel foil (Hanser, 2011). In a surface barrier detector, a depletion region is formed at the surface of an $n$-type silicon crystal by evaporating a thin gold $(\mathrm{Au})$ layer onto one surface after etching (Knoll, 2000, p. 377). An aluminum layer on the other surface forms an ohmic contact (Coleman et al., 1968a, 1968b). The outer collimator defines the $30^{\circ}$ full-width of the cylindrical FOV while the inner collimator defines the $13^{\circ}$ full-width flat maximum of the angular response function, resulting in an effective solid angle of $0.112 \mathrm{sr}$ (Hanser, 2011). A $0.76 \mu \mathrm{m}$ nickel foil between the detector and the inner collimator provides solar blindness (Hanser, 2011). (In the hours around local noon, several telescopes look directly at the sun). This foil also stops lower-energy protons from reaching the detector; this cutoff energy is $135 \mathrm{keV}$ (Galand \& Evans, 2000) for the SEM-1 MEPED, which had a 0.696 or $0.511 \mu \mathrm{m}$ nickel foil (Seale \& Bushnell, 1987). The MAGED and MEPED electron telescope designs are similar. The MAGED and MEPED electron channels are determined by logic on-orbit, but the logic is different for MAGED, producing five quasi-differential channels (Hanser, 2011; Table 1).

\subsection{MAGPD}

Each MAGPD telescope consists of two 200- $\mu$ m surfacebarrier silicon detectors located behind two collimators and a sweep magnet (Hanser, 2011). The angular response is similar to that of MAGED. The sweep magnet prevents electrons below

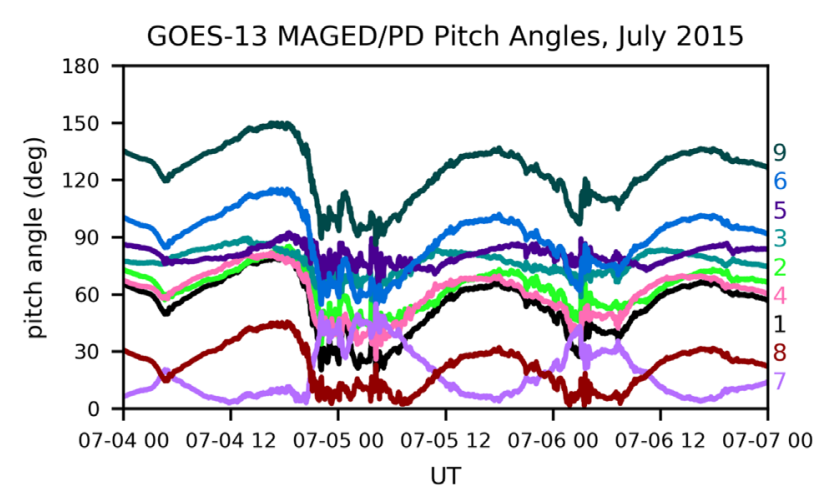

Fig. 1. Three days of GOES-13 MAGED and MAGPD telescope pitch angles (4-6 July 2015), showing examples of disturbed and quiet periods. The pitch angles are at the centers of the telescope FOVs. The telescope numbers are identified at the right, ordered by the pitch angle values at 00 UT on 7 July 2015, using colors corresponding to those of the traces.

several hundred $\mathrm{keV}$ from reaching the detectors (Hanser, 2011). The front detector has a $120 \mu \mathrm{g} \mathrm{cm}^{-2}(0.444 \mu \mathrm{m})$ $\mathrm{Al}$ coating facing outward to exclude solar photons and to provide the ohmic electrical contact (Hanser, 2011). The rear detector has a $40 \mu \mathrm{g} \mathrm{cm}^{-2}(0.021 \mu \mathrm{m})$ Au surface barrier contact facing forward (Seale \& Bushnell, 1987). These features are common to the MAGPD and MEPED proton telescopes, except that the $\mathrm{Al}$ coating on the front MEPED detector is $0.074 \mu \mathrm{m}$ thick $\left(20 \mu \mathrm{g} \mathrm{cm}^{-2}\right.$ ) (Evans \& Greer, 2000; Panametrics, 2004b). By virtue of this thinner coating, MEPED can detect protons down to $30 \mathrm{keV}$ but is light-sensitive - not known to be a problem given the geometry of the instrument look directions and of the orbital planes in which MEPED flies (Panametrics, 2004b). MAGPD and MEPED use different on-orbit logic based on discrete thresholds to form the proton channels. In MEPED, the rear detector is used in coincidence to define a $>6.9 \mathrm{MeV}$ channel as well as in anti-coincidence to define the lower-energy channels (Evans \& Greer, 2000). In MAGPD it is used only in anti-coincidence to produce five quasi-differential channels (Hanser, 2011; Table 1). The anticoincidence between the front and rear detectors prevents $\mathrm{MeV}$ protons that enter through the collimators and deposit the same energy in the front detector as a $\mathrm{keV}$ proton from contaminating the 10 's of $\mathrm{keV}$ channels.

\subsection{MPA}

The magnetospheric plasma analyzer (MPA) consists of an electrostatic analyzer connected to an array of six channel 
MPA channels $0-1$ and MAGED ME1 bandpasses

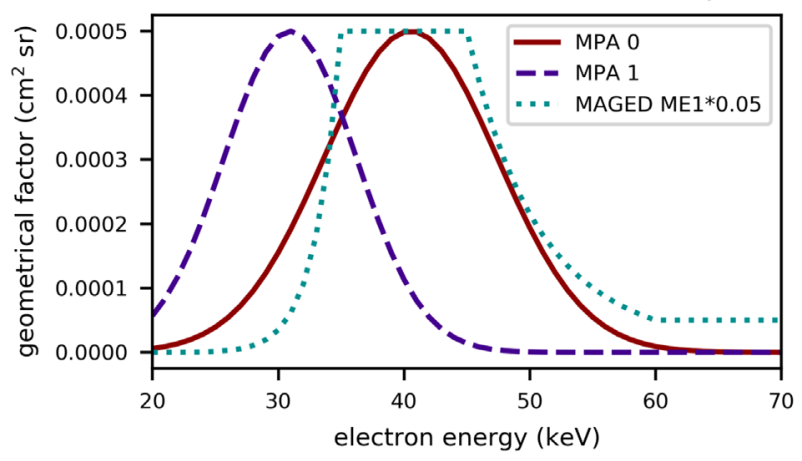

Fig. 2. Comparison of energy responses of the two highest-energy MPA electron channels ( 0 and 1$)$ and the lowest-energy MAGED channel (ME1). The ME1 geometrical factor is scaled by 0.05 .

electron multipliers that observes electrons and ions alternately to produce a three-dimension distribution on a spinning satellite (Bame et al., 1993; Thomsen et al., 1999). It was first launched in 1989 and has flown on multiple satellites in geosynchronous orbit. The energy range is approximately $1 \mathrm{eV} / \mathrm{q}-40 \mathrm{keV} / \mathrm{q}$. The normalized full-width half maximum of the analyzer energy response $\mathrm{d} E / E$ is 0.4 and the peak geometrical factor is $\sim 5 \mathrm{e}-4 \mathrm{~cm}^{2}$ sr (Bame et al., 1993). The 1991-080 channel energy values (mid-range among the five instruments listed in Table 1 of Thomsen et al., 1999) for electron channel 0 are 46,375$35,412 \mathrm{eV}$ and for electron channel 1 are 35,412-27,041 eV. Using these values and modeling the MPA responses as Gaussians, the energy responses of MPA electron channels 0 and 1 and MAGED channel ME1 are plotted in Figure 2. This comparison shows that MAGED ME1 and MPA electron channel 0 observe a similar range of electron energies. This energy overlap permits a direct comparison of electron observations by the two instruments. The MPAs chosen for comparison with MAGED and MAGPD were on the satellites closest to GOES-13 and -15 . The satellite closest to GOES-13 $\left(\sim 75^{\circ} \mathrm{W}\right.$ during the entire period) was 1994-084 $\left(\sim 49^{\circ} \mathrm{W}\right)$, and the satellite closest to GOES-15 $\left(\sim 135^{\circ} \mathrm{W}\right.$ during most of the period) was LANL-01A $\left(\sim 165^{\circ} \mathrm{W}\right)$.

\section{Comparison of GOES 13, 14 and 15 after GOES-15 launch}

A particular strength of the MEPED correction method of Sandanger et al. (2015) was the use of multiple satellite overlaps to quantify the degradation of earlier models. At the time of that paper, seven SEM-2 MEPEDs had been launched, and observations during the first years of the four newer models were used to correct older models flying at approximately the same local times in the polar regions. Unfortunately, circumstances made this technique not as useful with GOES 13-15. Only three pairs of MAGEDs and MAGPDs were launched, and when GOES-14 turned on in July 2009, GOES-13 was not returning data to SWPC. Therefore, the only opportunity to compare a newly-launched MAGED and MAGPD pair with older models presented itself in 2010 after the launch of GOES-15. From
May 2010 to September 2011, GOES-15 was at $\sim 90^{\circ} \mathrm{W}$ and separated one hour in local time from GOES-13 $\left(\sim 75^{\circ} \mathrm{W}\right)$ and GOES-14 $\left(\sim 105^{\circ} \mathrm{W}\right)$. At such separations, with the satellites at similar geomagnetic latitudes, cross-calibrations have been performed successfully between GOES $>2 \mathrm{MeV}$ electron observations (Onsager et al., 2004; Meredith et al., 2015).

To compare the MAGED and MAGPD observations from the three satellites during this period, we performed statistical asynchronous regressions (SAR) (O'Brien et al., 2001) between GOES-15 and GOES-13 and -14 observations. SAR was used because, although the three satellites were in relatively close proximity, they did not observe fluxes simultaneously at the same local time. SAR has been used to relate geosynchronous $\mathrm{MeV}$ electron fluxes observed at different local times (O'Brien et al., 2001; O'Brien \& McPherron, 2003; Burin des Roziers \& $\mathrm{Li}, 2006)$. July 2010 was chosen because it was one of the two months during May-September 2010 with the lowest mean daily $A p$ index (5), ensuring that the cross-calibration was performed when geomagnetic activity was minimized. In July 2010, GOES-13, -14, and -15 had been on-orbit 4 years, 1 year, and 4 months, respectively. The time series from this month in the lowest-energy electron and proton channels from the three satellites are shown in Figure 3. The $40-\mathrm{keV}$ electron fluxes exhibit order-of-magnitude increases due to injections of plasma sheet electrons (on open drift paths), interspersed with days-long flux decreases due to losses (on closed drift paths). The approximate $40-\mathrm{keV}$ electron flux limit between the two regimes is $\sim 4 \times 10^{4} \mathrm{~cm}^{-2} \mathrm{sr}^{-1} \mathrm{keV}^{-1} \mathrm{~s}^{-1}$. At these energies, access of plasma sheet electrons to geosynchronous orbit (via injections) is well correlated with $K p>2$ (Korth et al., 1999). This behavior is characteristic of electric-field-dominated drifts. Below $\sim 2 \times 10^{3} \mathrm{~cm}^{-2} \mathrm{sr}^{-1} \mathrm{keV}^{-1} \mathrm{~s}^{-1}$, the $40-\mathrm{keV}$ electron flux corresponds to dropouts (for example, during the substorm growth phase prior to an injection). In contrast, the 95-keV proton fluxes exhibit a diurnal variation characteristic of geostationary orbit that results from the generally noncircular nature of gradient-curvature drift paths (Sibeck et al., 1987). Both the electrons and protons exhibit deep drop-outs while the proton injections are not as large as the electron injections.

The agreement among the electron measurements from the three satellites is excellent (apart from during injections and dropouts, probably due to local time differences), while the proton fluxes from GOES-13 are noticeably lower than those from the other two satellites. This is reflected in the complementary cumulative distribution functions (CDFs) shown in Figure 4. The logarithms of the complementary CDFs (1-CDF) are plotted versus the square root of fluxes, following O'Brien et al. (2001). (On such a plot, an exponential function of the square root of flux is a straight line). The two dashed vertical lines on the 40-keV electron plot correspond to the flux levels of $2 \times 10^{3}$ and $4 \times 10^{4} \mathrm{~cm}^{-2} \mathrm{sr}^{-1} \mathrm{keV}^{-1} \mathrm{~s}^{-1}$ discussed above. In the regime between the dashed vertical lines, which corresponds to closed drift paths, the complementary CDFs for the three satellites agree very well. To the right of the second vertical line, in the open drift path (plasma sheet access) regime, the distributions agree less well but are still quite similar. The disagreement among the proton distributions is much greater. The GOES-13 proton distribution departs substantially from those from GOES-14 and -15, as expected from the time series 

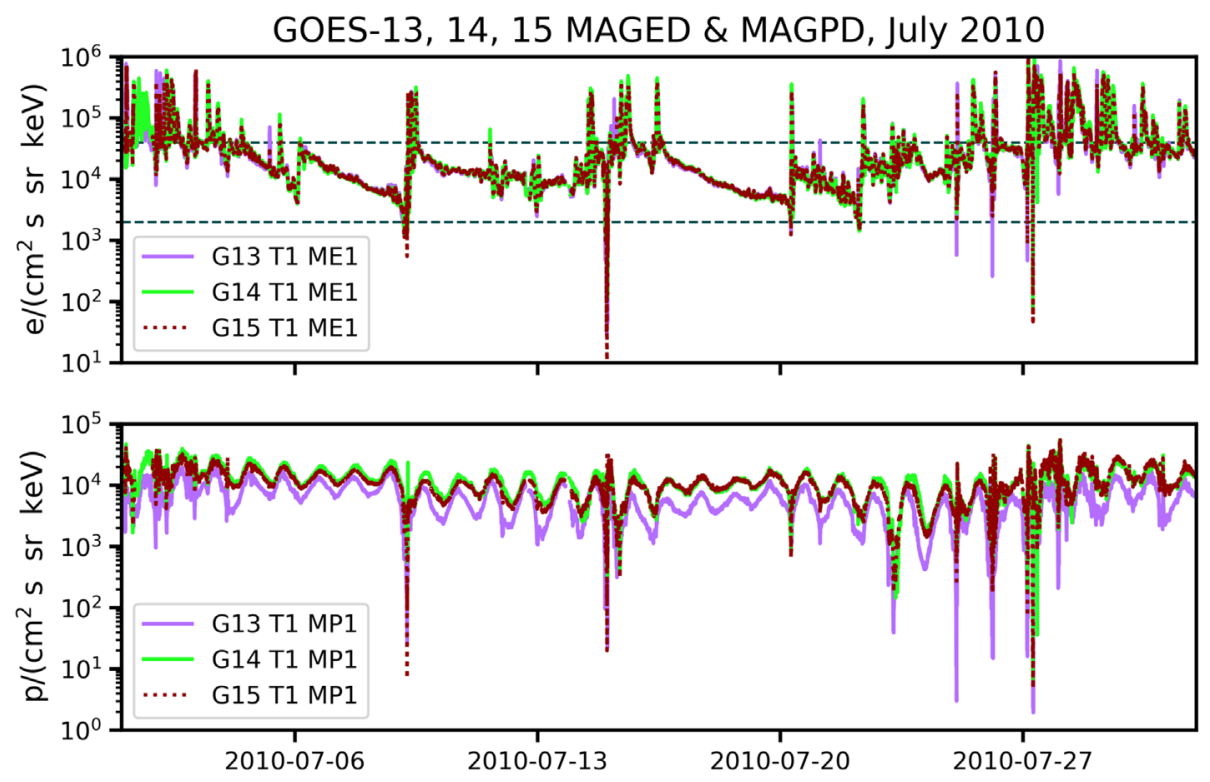

Fig. 3. (Upper panel) Electron fluxes and (lower panel) proton fluxes from GOES-13, 14 and 15 during July 2010, from Telescope 1 and the lowest energy channel ( $40 \mathrm{keV}$ electrons, $95 \mathrm{keV}$ protons). The upper dashed line indicates the approximate limit between plasma sheet electron fluxes on open drift paths (above) and fluxes on closed drift paths (below). The lower dashed line indicates the level below which the fluxes correspond to dropouts.
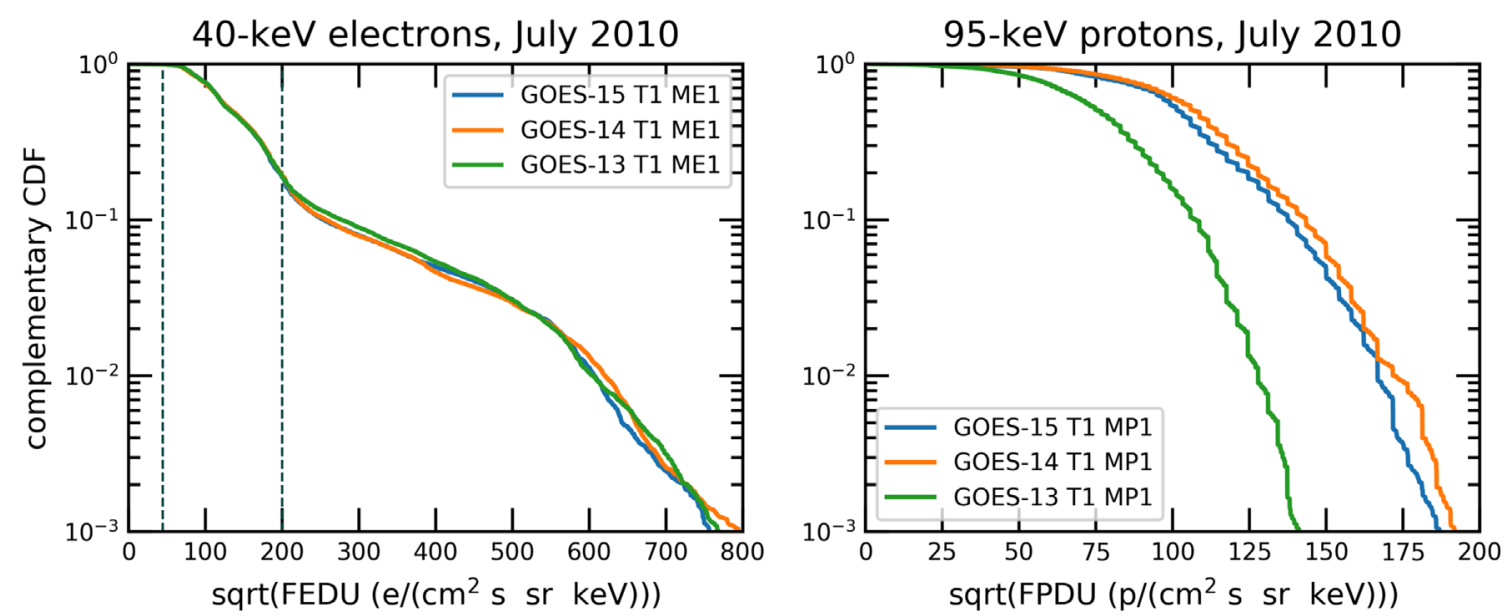

Fig. 4. Complementary CDFs (1-CDF) of GOES 13-15 Telescope 1 40-keV electrons (ME1) and 95-keV protons (MP1) during July 2010, plotted versus the square root of flux. The vertical dashed lines on the left-hand plot are discussed in the text.

plot (Fig. 3). The GOES-14 proton fluxes reach slightly greater extremes than those observed by GOES-15, possibly due to calibration differences or physical differences between the two locations.

The regression results are shown in Figure 5 for the five MAGED channels and the five MAGPD channels (from Telescope 1) during July 2010 . The results are relatively smooth because the SAR is calculated by comparing cumulative distribution functions (CDFs). A Theil-Sen fit (e.g., Sen, 1968) is performed to the base-10 logarithm of fluxes so that the highest fluxes do not overly dominate the result. In order to interpret the results, consider that the linear $(A 1)$ and constant $(A 0)$ coefficients from the fit relate the two satellite fluxes as $j 13$ or $j 14=10^{A 0} j 15^{A 1}$. If $A 1$ is close to unity, then the comparison gives essentially a linear relationship between the two fluxes. This indicates ideal conditions for a cross calibration, because the instrument responses to fluxes are linear over several orders of magnitude, and a non-paralyzable dead-time correction has been applied. A significant departure of $A 1$ from unity indicates that factors other than a simple calibration difference are affecting the result, such as different behaviors at different particle energies.

The ME1 comparison is an example of excellent agreement among the three satellites despite different periods on-orbit. The two pairs of dashed lines on the $40-\mathrm{keV}$ electron plot correspond to the flux levels of $2 \times 10^{3}$ and 

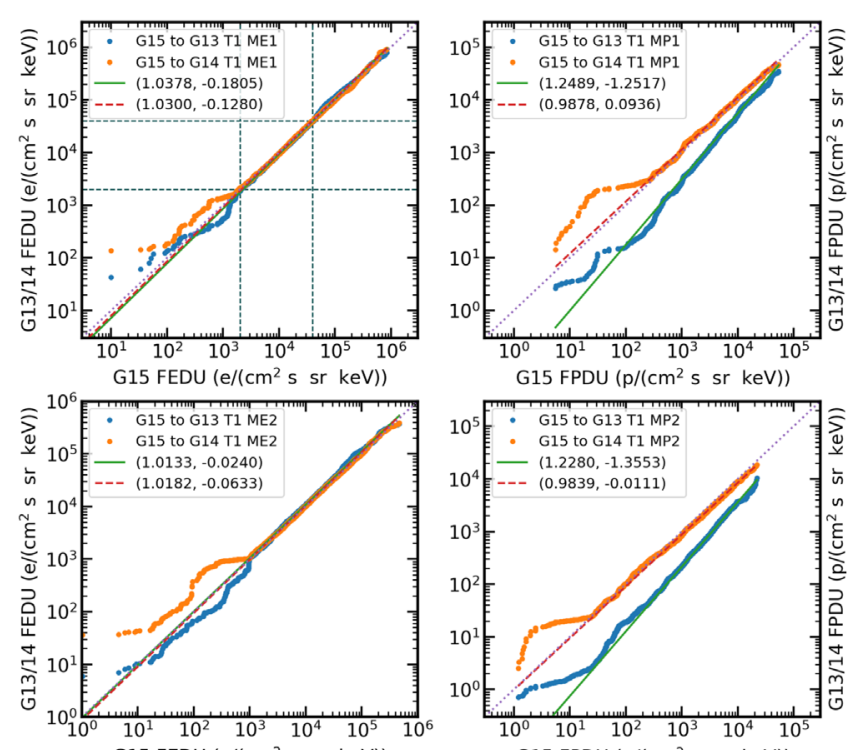

G15 FPDU (p/( $\mathrm{cm}^{2}$ s sr keV))

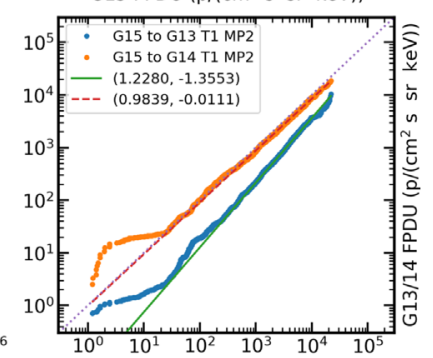

G15 FEDU $\left(\mathrm{e} /\left(\mathrm{cm}^{2} \mathrm{~s}\right.\right.$ sr $\left.\left.\mathrm{keV}\right)\right)$

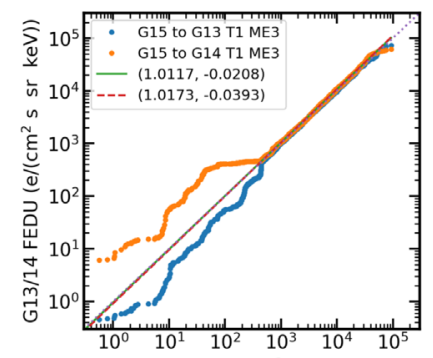

G15 FEDU (e/( $\mathrm{cm}^{2} \mathrm{~s}$ sr $\left.\left.\mathrm{keV}\right)\right)$

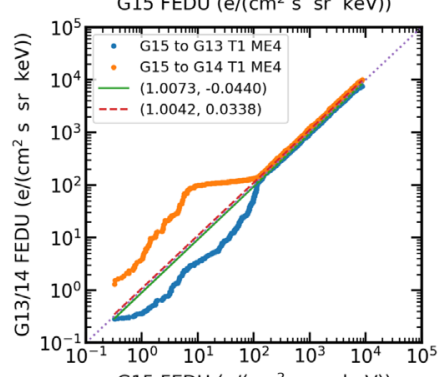

G15 FEDU (e/( $\mathrm{cm}^{2} \mathrm{~s}$ sr $\left.\left.\mathrm{keV}\right)\right)$
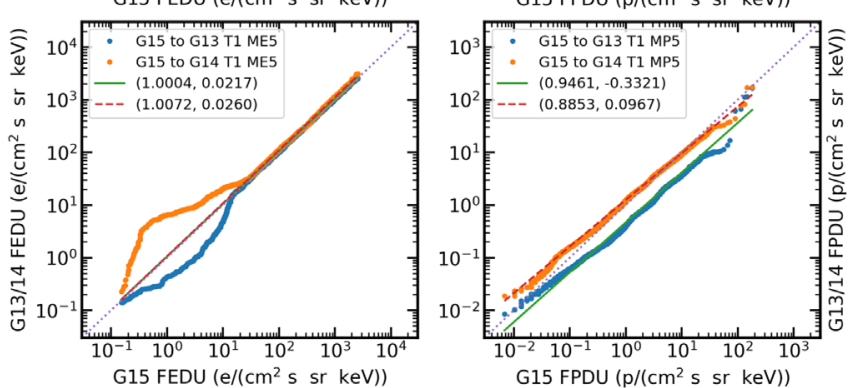

Fig. 5. Statistical asynchronous regressions (SAR) of GOES-15 mapped to GOES-13 and -14 particle fluxes during July 2010. The left column shows the results for the MAGED Telescope 1 ME1ME5 channels. The right column shows the results for the MAGPD Telescope 1 MP1-MP5 channels. The dotted diagonal line represents the identity relationship. The results of the Theil-Sen fits to the mappings are described in the legends by a pair of numbers representing the first- and zeroth-order terms of a linear fit to $\log 10$ (flux). FEDU $=$ differential unidirectional electron flux. FPDU $=$ differential unidirectional proton flux.
$4 \times 10^{4} \mathrm{~cm}^{-2} \mathrm{sr}^{-1} \mathrm{keV}^{-1} \mathrm{~s}^{-1}$ discussed above. Fluxes below $\sim 2 \times 10^{3} \mathrm{~cm}^{-2} \mathrm{sr}^{-1} \mathrm{keV}^{-1} \mathrm{~s}^{-1}$ correspond to dropouts, during which local time differences are expected. Above that level, agreement is excellent, indicating that the ME1 fluxes are stable despite (in the case of GOES-13) being on-orbit for four years. Small variations above $4 \times 10^{4} \mathrm{~cm}^{-2} \mathrm{sr}^{-1} \mathrm{keV}^{-1} \mathrm{~s}^{-1}$ correspond to particle injections, again where local time differences are expected. The pattern is similar in the higher energy electron channels; the pronounced differences at the lowest flux levels correspond to dropouts. In contrast to the electron fluxes, the GOES-13 proton fluxes differ greatly from GOES-15 in all five energy channels. In addition, the MP1 and MP2 relationships have power law exponents that are far from unity, resulting in a significant flux-dependent discrepancy. The GOES-14 and 15 fluxes agree much better, the agreement being best for MP1 and MP2. As with the electron channels, the departures from straight lines at the lowest proton fluxes are attributed to natural flux dropouts.

This comparison shows that some effect other than linear calibration offsets caused large discrepancies between the GOES-15 and -13 MAGPD fluxes during this period in 2010 soon after the launch of GOES-15. Based on prior experience with the similar MEPED proton telescopes, it is likely that radiation damage had caused the GOES-13 MAGPD to degrade in the four years since launch. In contrast, there was no clear indication that GOES-14 MAGPD had degraded after a year on orbit. The MAGPD degradation evident in July 2010 indicates that preparing the proton fluxes for ingest into the MPA-based model requires correcting for such degradation over the period of interest. Indeed, three of the five steps in the next section provide a partial correction for the long-term degradation of MAGPD.

\section{Correction and cross-calibration methods and results}

The correction and cross-calibration process followed five steps, which are described here in the order in which they were applied. First, noise bursts and other spurious features were removed from the fluxes and magnetic field data. This step was necessary to perform first so that the spurious features did not influence the cross-calibrations. Second, the nine telescopes of each MAGED and MAGPD were cross-calibrated, a process referred to as "intra-calibration" since these comparisons were performed within an instrument. At this point, the individual cleaned telescope fluxes were scaled by the results of the intra-calibration and combined to determine the omnidirectionally-averaged fluxes. These are necessary for ingest into the target model, which is derived from spin-averaged MPA fluxes. The fourth step was to cross-calibrate the GOES-13 and -15 omnidirectional fluxes at a time when the GOES-15 detectors had been on-orbit less than a year and therefore had not exhibited significant degradation This step was essential to correct the GOES-13 MAGPD fluxes for the degradation suffered from launch through 2010. Finally, the GOES and LANL MPA data were cross-calibrated in order to ensure consistency in the target model. In the following sections, the method for each step is described and the results are presented before proceeding to the next step. 


\subsection{Data cleaning}

Spurious signals needed to be removed from the telescope fluxes before they could be used in cross-calibrations or in calculating omnidirectional flux. The signatures of in-flight calibrations were already removed by the real-time ground processing. Several of the surface-barrier detectors used in the GOES 13-15 MAGED and MAGPD telescopes exhibited excessive noise during the first several months post-launch. For example, at launch, GOES-14 MAGPD Telescope 5 (T5) had a noisy rear detector, which, since it operates in anti-coincidence with the front detector, reduced the coincidence count rates by a factor of 10-20. This problem disappeared on 28 February 2010, resulting in an abrupt upward jump in the T5 fluxes.

Other detectors developed noise after some time on-orbit. The GOES-15 MAGED Telescope 2 (T2) data had to be omitted entirely from the analysis due to severe noise that first manifested itself in June 2012. In GOES-13 MAGED T6 fluxes, discrete noise bursts repeatable in local time started appearing around the June solstice starting in 2012. In GOES-15 MAGED $\mathrm{T} 1$, similar noise bursts started occurring around the December 2016 solstice (but not around the June solstice). These noise bursts were typically an order-of-magnitude larger than the natural electron fluxes. In order to salvage the GOES-13 T6 and GOES-15 T1 electron data for the entire period of interest, a method was developed to remove these discrete noise bursts. Taking the latter as an example, superposed epoch analysis revealed that the $\mathrm{T} 1$ noise bursts repeatedly lasted $\sim 3.5 \mathrm{~h}$ and were centered $\sim 2.5 \mathrm{~h}$ past local noon. This permitted their identification and removal based on solely on calendar date and time stamp. The result is illustrated in Figure 6. This time series from November 2017 through January 2018 illustrates how the T1 noise bursts commence about a month before and subside about a month after December solstice. The orange traces indicate the noise bursts (removed) and the purple traces represent the resulting cleaned time series.

A separate problem involved the GOES-15 magnetometer data, used to calculate pitch angles for the calculation of omnidirectional flux. There were eight periods (between June 2015 and September 2018) during which the primary GOES-15 magnetometer exhibited strongly anomalous behavior. The anomalous periods lasted from several hours to several days. The cause of this problem has not yet been determined (R. Redmon, priv. commun.). These periods have been omitted from the calculation of the GOES-15 omnidirectional fluxes.

\subsection{Intra-calibration of MAGED and MAGPD telescopes}

The real-time ground processing of the MAGED and MAGPD data uses the design values of the channel energy ranges and geometrical factors to characterize the channels. The design values were validated by beam testing of a single engineering-model telescope (Panametrics, 2004a, 2004b). Therefore, variations among telescopes, both pre- and postlaunch, are not accounted for in the real-time ground processing. For the present reprocessing effort, the variations among telescopes were intra-calibrated following the method of Rowland \& Weigel (2012). This method is based on the recognition that fluxes of the same energy observed by two separate telescopes at the same local pitch angle should be the same, assuming the fluxes are uniform in gyro phase. Rowland \& Weigel (2012) applied this method to two 5-month periods of MAGED data. The present work represents the first time this method has been applied to MAGPD data, the first time it has been used to analyze multiple years of data, and the first time the resulting scale factors (SFs) have been used to improve the accuracy of a product.

The analysis used one-minute flux averages and pitch angles calculated from 1-min averages of the magnetic field. First, pitch angles $\alpha$ greater than $90^{\circ}$ were replaced by their supplementary angles $\left(180^{\circ}-\alpha\right)$. This assumes that, on average, the upward fluxes $\left(\alpha>90^{\circ}\right.$ since GOES lies north of the geomagnetic equator) are the same as the downward fluxes $\left(\alpha<90^{\circ}\right)$. This assumption is necessary in order to have a sufficient ( $>100$ ) and non-zero number of comparisons for all telescope pairs (Fig. 1). There is also an implicit assumption that the fluxes are uniform in gyro phase. Second, a pitch angle match was identified if the pitch angles of the two telescopes agreed to within a tolerance of $1^{\circ}$ and if the pitch angle change in one minute was less than $30^{\circ}$. (This criterion applies to all energy channels since pitch angles are the same for all energy channels). Third, for a given energy channel, a pitch angle match was rejected if one or both telescopes registered fewer than 100 counts in $1 \mathrm{~min}$.

The Nelder \& Mead (1965) non-linear minimization technique (from scipy.optimize.minimize) was used to minimize the objective function, which is the sum of the squares of the following relative difference calculated for each of the matches $m$ in a three-month period:

$$
R(m)=\frac{\mathrm{SF}_{i}(m) j_{i}(m)-\mathrm{SF}_{j}(m) j_{j}(m)}{\mathrm{SF}_{i}(m) j_{i}(m)+\mathrm{SF}_{j}(m) j_{j}(m)}
$$

where $\mathrm{SF}_{i}$ is the multiplicative scale factor for telescope $i$ and $j_{i}$ is the flux measured by telescope $i$. The maximum number of iterations was set to 2000, but this limit was never reached. With the absolute tolerance on the objective function set to 0.01 and the absolute tolerance on the solutions (SFs) set to 0.001 , the number of iterations per channel was typically in the range 300-800. A moving three-month analysis period was chosen in order to increase the number of matches and to smooth the results somewhat. For GOES-13 MAGPD, the number of matches in three-month periods starting January 2011 ranged from 173 (T7 MP5, January 2013) to 66,811 (T4 MP1, January 2011). This meets the criterion suggested by Rowland \& Weigel (2012) of a lower bound of 30 to the number of acceptable matches.

These SFs are relative and therefore need to be normalized to the SFs for a single telescope. This telescope was chosen for relatively low scatter and long-term variability in its SFs, and typical non-storm pitch angles between $45^{\circ}$ and $135^{\circ}$. For MAGED, the normalizing telescopes were 3 (GOES-13) and 5 (GOES-15). For MAGPD, the normalizing telescopes were 1 (GOES-13) and 4 (GOES-15). The intra-calibration SFs correct for short-term variations as well as long-term drift relative to the normalizing telescope, including the MAGPD degradation identified in Section 3. The behavior of the normalizing telescope thus determines comparisons with other instruments.

The normalized SFs for GOES-13 and -15 are shown in Figures 7 and 8. The GOES-13 MAGED normalized SFs are smoothly varying, ranging from 0.82 to 1.05 . The clearest signal 


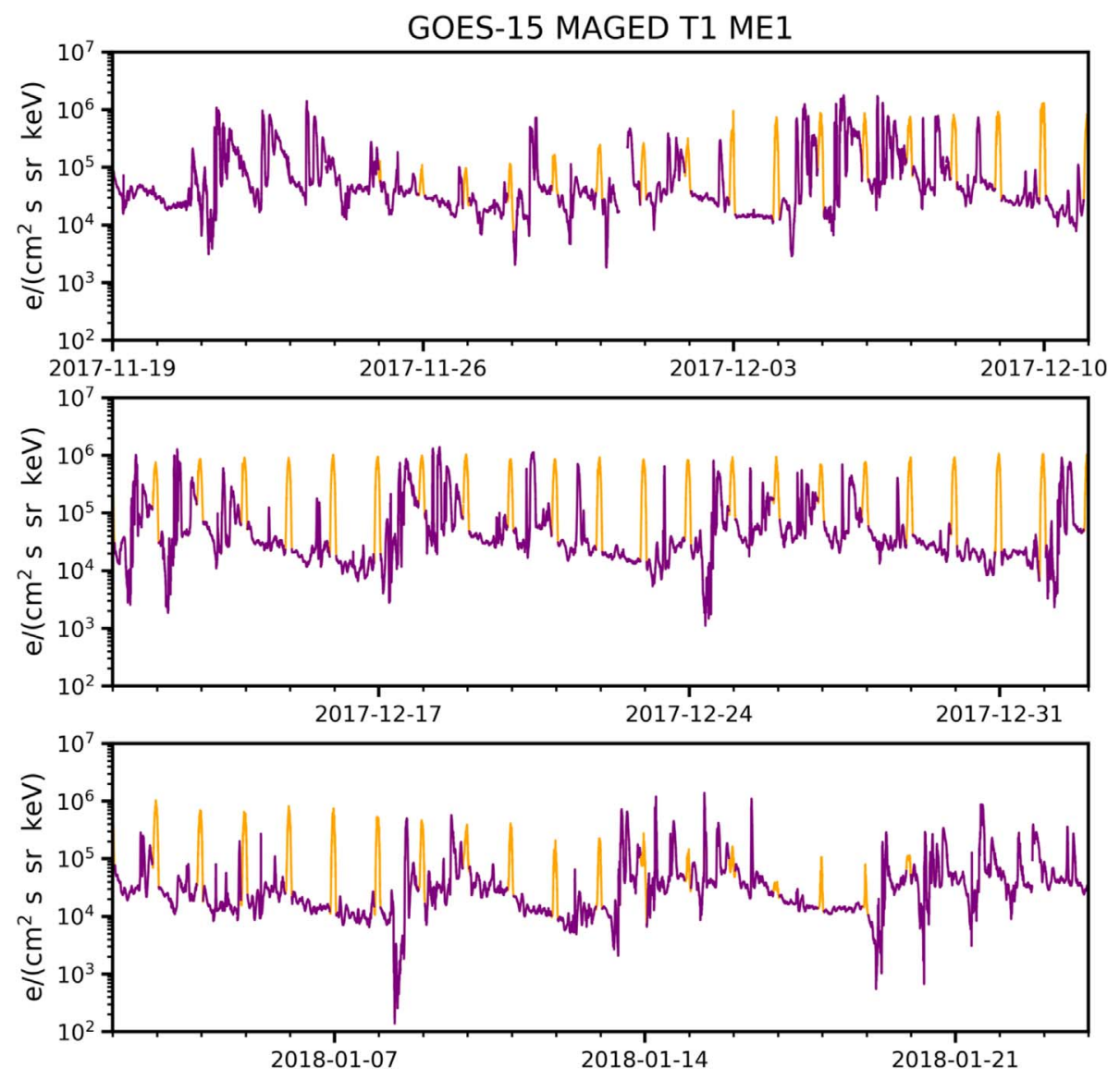

Fig. 6. GOES-15 MAGED Telescope 1 ME1 channel (40 keV electrons), 19 November 2017-23 January 2018. The orange traces represent the noise bursts removed from the data.

is an annual variation most pronounced in T7, T8, and T9. The SF minima were around the December solstice, the deepest variation occurring around the December 2017 solstice (indicating that the fluxes in these telescopes were artificially high). The GOES-13 MAGPD SFs exhibit more complex behavior, ranging from 0.48 to 1.8 . There is an annual variation in some telescopes (most notably T2) but the SFs tend to peak rather than dip around the December solstice. There are shorter-term variations in T7-T9. Long-term drifts are also clear, most notably in T2-T6.

Compared to GOES-13, the GOES-15 SFs exhibit more scatter and deeper annual variations. (The GOES-13 T2 was omitted from the calculation of SFs due to the severe noise problem noted above). The GOES-15 MAGED SFs range from $>0.65$ to $<1.15$. An annual variation is most clear in T1. Shorterterm variations are observed around the December solstices, particularly T6-T8. (The solstitial noise bursts described above were removed from GOES-15 T1 and from GOES-13 T6 before the calculation of SFs, and there were no noise bursts in the other telescopes). The GOES-15 MAGPD normalized SFs exhibit even greater variation, ranging from 0.19 to 2.8 . In some telescope/channel combinations, the cycle peaks around the December solstice while in others it reaches a minimum.

This analysis implicitly assumes that the field orientation observed by the GOES magnetometers is without error. It is possible that some of the observed variation is due to the magnetometers. However, the pitch angles for all MAGED and MAGPD telescopes are calculated from a common set of field vectors. Therefore, given the wide range of behaviors observed among the MAGED and MAGPD telescopes and channels on each satellite, it is more likely (and indeed almost certain) that the dominant variations are due to the behavior of the individual telescopes.

\subsection{Calculation of omnidirectionally-averaged fluxes}

For the target model, omnidirectional averages of the MAGED and MAGPD fluxes were calculated using the GOES magnetic field to calculate time-varying pitch angles. The formulation for this calculation has been described by Sillanpää et al. (2017) and Ganushkina et al. (2019). The key difference with respect to the earlier calculations was the application of the intercalibration scale factors from the previous section to the telescope fluxes prior to the numerical integration over pitch angle. The scale factors were assigned to the center of the threemonth periods over which they were calculated and interpolated to the time stamp of each 1-min average using a second-order polynomial in time. Periods of contaminated fluxes or magnetic fields (described in Sect. 4.1) as well as periods of satellite yaw flips were omitted from this calculation. The resulting averages have the same units as differential unidirectional flux (particles $\mathrm{cm}^{-2} \mathrm{sr}^{-1} \mathrm{keV}^{-1} \mathrm{~s}^{-1}$ ). 

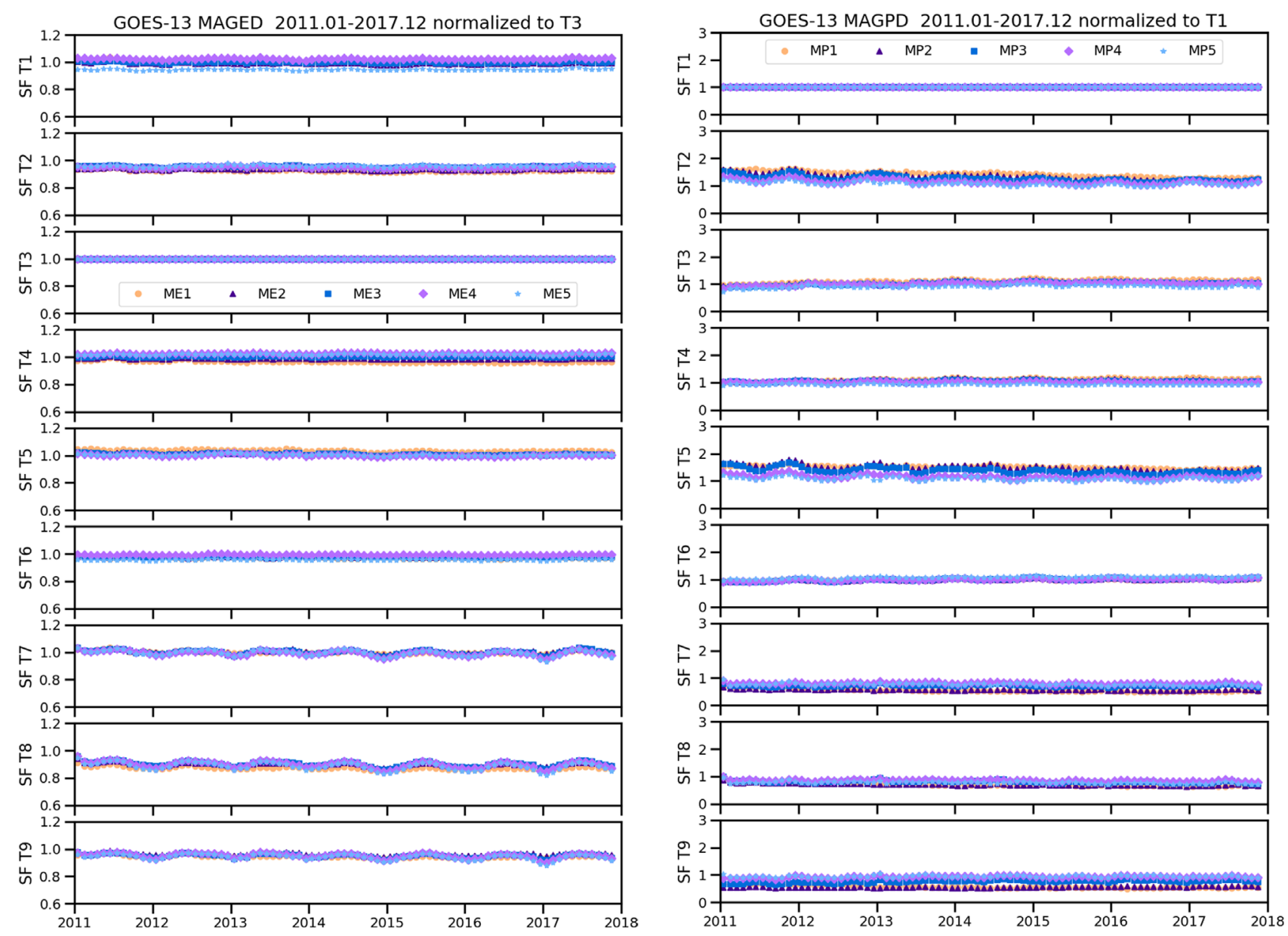

Fig. 7. Intra-calibration scale factors (SFs, unitless) for GOES-13 MAGED (left) and MAGPD (right), 2011-2017. Each panel illustrates the SFs for all five energy channels from one telescope.

\subsection{Cross-calibrations of GOES omnidirectional fluxes}

As discussed in Section 3, the period after the launch of GOES-15 presented the only opportunity to compare a newlylaunched MAGPD with others already on-orbit. GOES-13 was not returning data when GOES-14 was launched. Therefore, the method of Sandanger et al. (2015) could be applied this one time to correct MAGPD fluxes. This was done by taking the ratios of time-averages of the omnidirectionally-averaged GOES-15 and -13 MAGPD fluxes during Bartels rotation 2421 (2010.12.31-2011.01.26) and applying them to the GOES-13 fluxes as a "day 1" correction (see below). These ratios of GOES-15 to GOES-13 proton fluxes were 1.99, $2.81,2.69,2.00$, and 1.77, respectively for MP1-5. The assumption that the GOES-15 proton fluxes had not degraded significantly by January 2011 after 10 months on orbit is supported by similarity with the example shown in Section 3 of GOES14 in July 2010 after a year on orbit. (Lack of publicly-available GOES-15 magnetometer data before January 2011 prevents an earlier cross-calibration between the omnidirectional fluxes).

\subsection{Comparisons of GOES and LANL MPA fluxes}

Bartels 27-day solar rotations were chosen for the averaging period as less arbitrary for magnetospheric quantities than calendar months. GOES-13 was at $\sim 75^{\circ} \mathrm{W}$; the LANL satellite closest to GOES-13 was 1994-084 ( 49 $\left.{ }^{\circ} \mathrm{W}\right)$. GOES-15 drifted from $\sim 90^{\circ} \mathrm{W}$ to $\sim 135^{\circ} \mathrm{W}$ in October-December 2011; the LANL satellite closest to GOES-15 was LANL-01A $\left(\sim 165^{\circ} \mathrm{W}\right)$. In order to compare the long-term variations of the LANL and GOES electron and ion fluxes, a second-order polynomial fit in time was performed on the 27-day flux averages. The intent of this fit order was to make the fewest assumptions about the long-term variation of the fluxes (short of a linear fit, which clearly would be inadequate).

In the case of MAGED and MPA $40 \mathrm{keV}$ electron fluxes (Fig. 9), the agreement is good over the long term despite the shorter-term variability, which is probably due in part to local time differences. The second-order fits to the $40 \mathrm{keV}$ fluxes are plotted in order to clarify the level of agreement. The worst-case discrepancy between these fits is $<20 \%$, at the beginning of 2011. The confidence in the MAGED $40 \mathrm{keV}$ electron fluxes achieved by this comparison transfers to the higher energy fluxes since they are measured by the same detector through the same aperture as the $40 \mathrm{keV}$ fluxes. Based on this comparison, we conclude that no correction needs to be applied to the MAGED fluxes in order to incorporate them accurately into the MPA-based geosynchronous statistical electron flux model. 

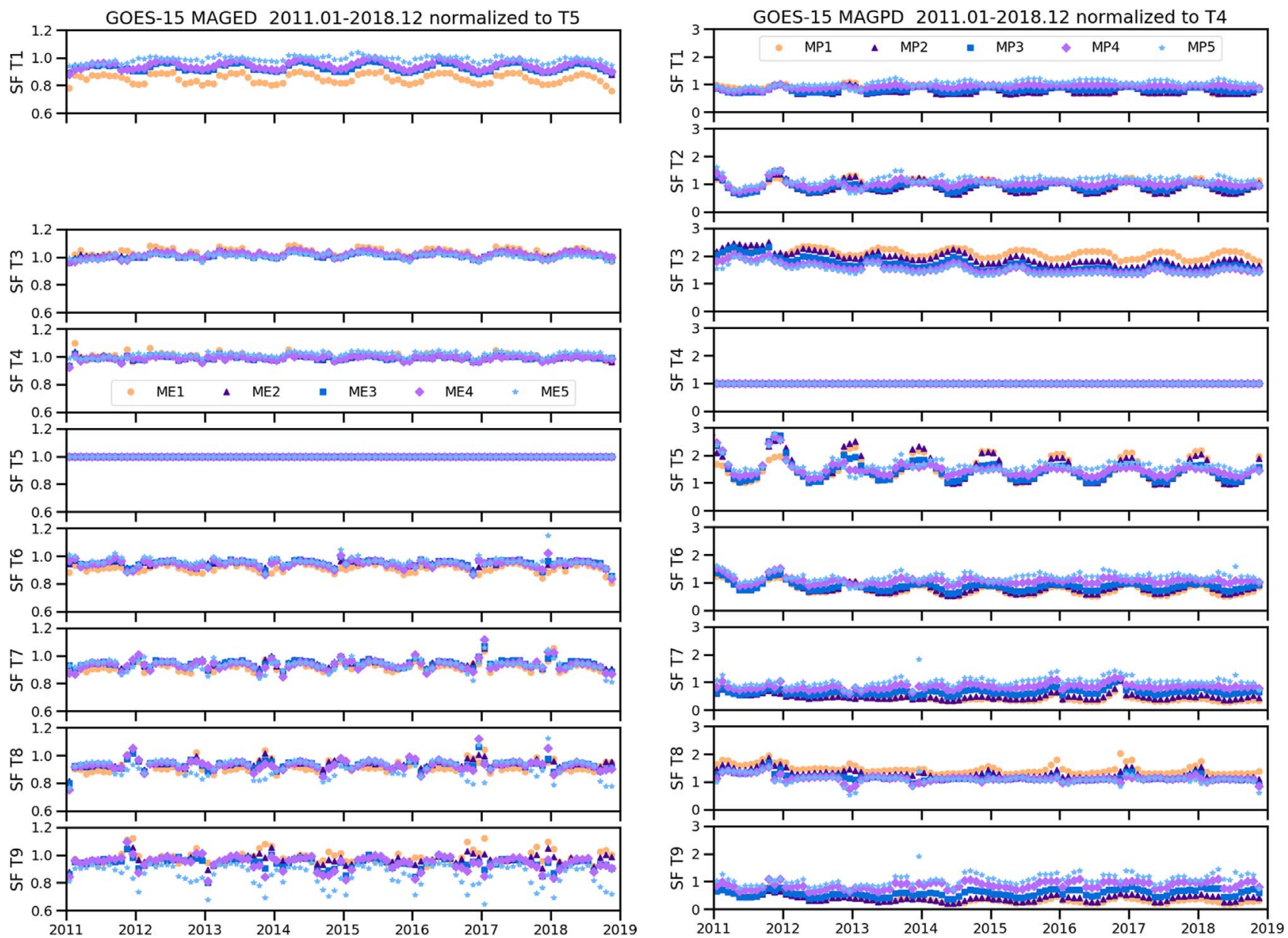

Fig. 8. Intra-calibration scale factors (SFs, unitless) for GOES-15 MAGED (left) and MAGPD (right), 2011-2018. Each panel illustrates the SFs for all five energy channels from one telescope. MAGED T2 was omitted due to severe noise.
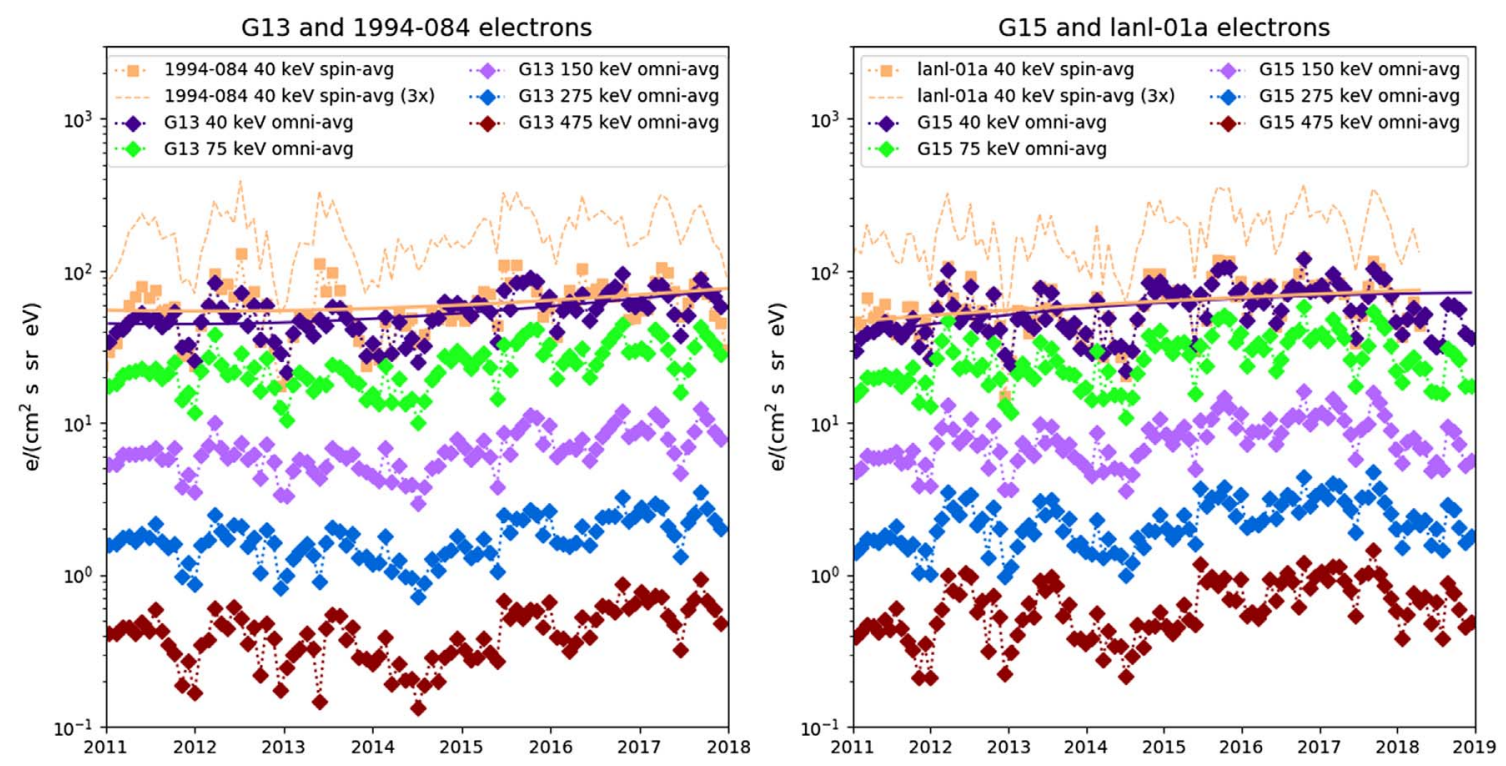

Fig. 9. Bartels-rotation averages of MPA $40 \mathrm{keV}$ electron and MAGED electron fluxes. (Left) GOES-13 and 1994-084 fluxes, $2011-2017$. (Right) GOES-15 and LANL-01A fluxes, 2011-2018. The second-order fits to the MPA and MAGED 40-keV electron fluxes are represented by solid lines. In each panel, the MPA fluxes are also plotted at three times their magnitude in order to distinguish them from the MAGED fluxes at the same energy. (The energy unit is converted from keV to eV to be consistent with the Denton et al., 2015, 2016 model). 

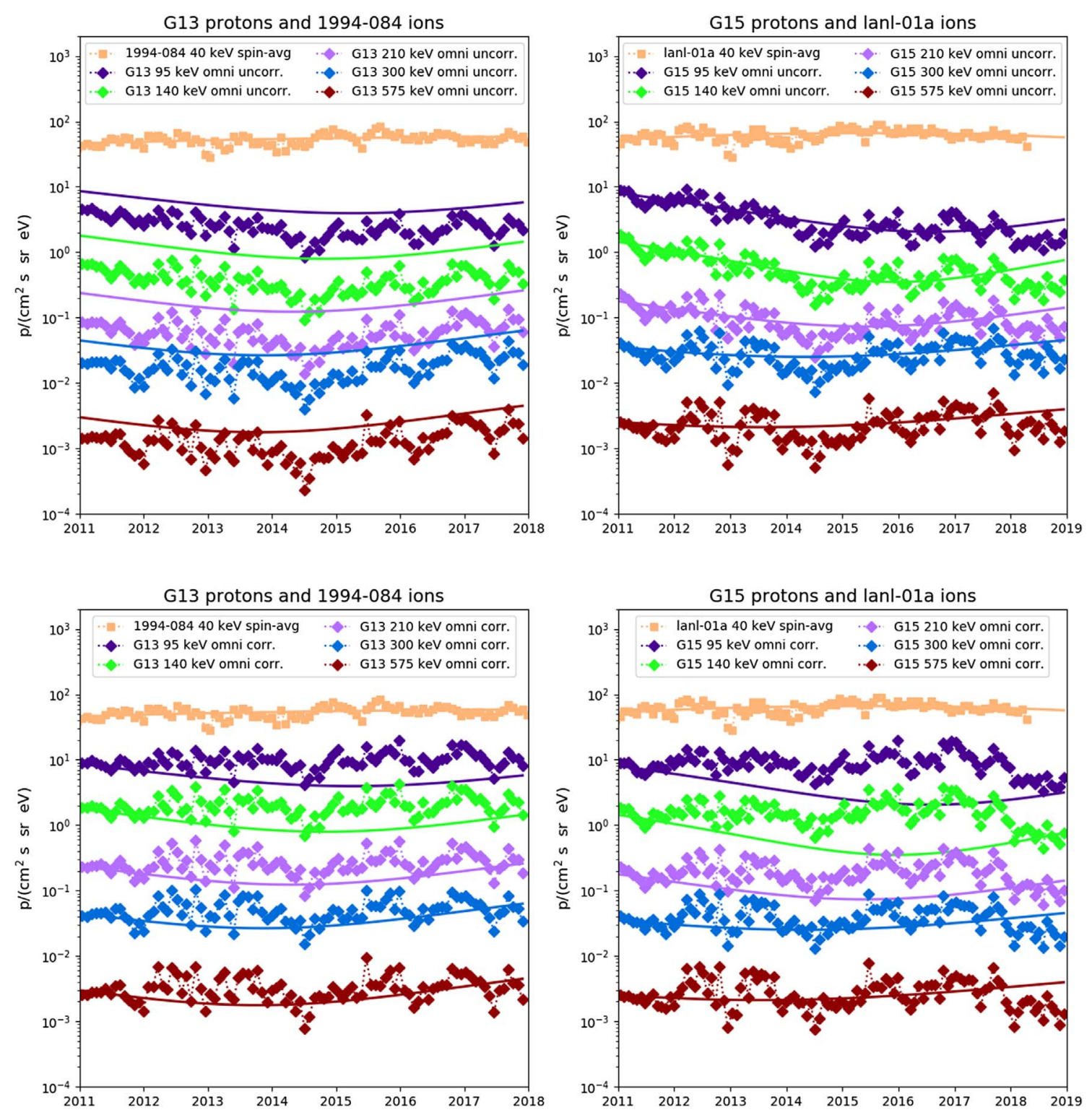

Fig. 10. Bartels-rotation averages of MPA $40 \mathrm{keV}$ ion and MAGPD proton fluxes, uncorrected (top) and corrected (bottom). (Left) GOES-13 and 1994-084 fluxes and fits, 2011-2017. (Right) GOES-15 and LANL-01A fluxes and fits, 2011-2018. The second-order fits to the fluxes are indicated by solid lines in both the uncorrected (top) and corrected (bottom) plots; the GOES-13 fits are to the data with the "day 1" correction applied. (The energy unit is converted from $\mathrm{keV}$ to $\mathrm{eV}$ to be consistent with the Denton et al., 2015, 2016 model).

In contrast, the MAGPD proton fluxes decline in amplitude over time (Fig. 10), in a way that is evident neither in the MPA $40 \mathrm{keV}$ ion fluxes nor in any of the electron fluxes from MPA or MAGED. This behavior indicates that the MAGPD telescopes suffer from long-term degradation on-orbit. In order to be incorporated into the MPA-based plasmasheet-radiation belt ion flux model, the MAGPD fluxes were corrected for this degradation via a cross-calibration with MPA that uses the second-order fits of flux versus time described above. The relative stability of the MAGED and MPA electron fluxes indicates that the MPA ion fluxes (measured by the same channel electron multipliers as the electron fluxes; Bame et al., 1993) provide a good basis for correcting the MAGPD fluxes. These fits are shown as solid smooth lines in Figure 10. In the case of GOES-13, the additional "day 1" corrections described above, derived from the comparison with the recently-launched GOES-15 when they were separated by an hour in local time, were applied prior to the fitting. The multiplicative correction applied to a given MAGPD channel was calculated from the ratio of the MPA fit to the MAGPD channel fit, normalized to the value on the first day (January 1, 2011).

The results show quantitatively that all GOES-13 MAGPD channels and the three lowest-energy GOES-15 channels degraded substantially over time. The two higher-energy GOES-15 channels were relatively flat. The rate of degradation was most rapid among the three lower-energy GOES-15 channels during the period of interest. The amount of degradation was sufficiently severe in the lowest energy GOES-15 channel 
(MP1, $95 \mathrm{keV}$ ) that its magnitude was similar to that of the next-higher energy channel corrected fluxes. The rate of degradation was similar among the five GOES-13 channels, though less rapid than among the three lower-energy GOES-15 channels. A significant "day 1" correction, as described above, was applied to the GOES-13 channels. However, the degradation did not continue during the entire period and even seemed to reverse itself in part. For example, in the case of GOES-15 MP1, the degradation appeared to cease after $\sim 6$ years on orbit, followed by a partial recovery. This behavior is not consistent with a model of degradation that is linear in cumulative fluence. (It should be noted that the GOES-14 rear detector noise issue that cured itself on 28 February 2010 resulted in a sudden factor-of-10 jump in coincidence count rates, which is distinct behavior from the long-term degradation observed here).

\section{Discussion}

The degradation of the MAGPD telescopes on-orbit is not surprising, given their similarity to the MEPED proton telescopes that have a well-known history of degradation on orbit. However, the evidence of a partial recovery of MAGPD response after 5-6 years on-orbit was unexpected, as was the stability of the MAGED telescopes, despite the fact that the nickel foil only keeps protons $<130 \mathrm{keV}$ from reaching the detector. The susceptibility of the MEPED proton telescope to on-orbit radiation damage was first reported by Lyons \& Evans (1984) and first investigated in depth by Galand \& Evans (2000) (see also Sect. 4.3.3 of Wüest et al., 2007). Galand \& Evans (2000) also demonstrated the stability of the MEPED electron telescopes. Subsequent work has been summarized well by Sandanger et al. (2015). The model used by Galand \& Evans (2000) and Sandanger et al. (2015) to explain the MEPED degradation is a combination of a dead layer and incomplete charge collection due to damage in the silicon, resulting in an underestimate of the incident particle energy.

A dead layer is a thin layer near the surface of a silicon detector in which ionization created by the particle being detected is incompletely or not at all collected, resulting in an underestimate of the energy deposited by the particle in the detector (Wuiest et al., 2007; Wall et al., 2014). Dead layers are created in surface barrier detectors by formation of the electrical contacts and by non-ionizing radiation damage that results in lattice defects (vacancies) that trap charge carriers (Knoll, 2000). While the name suggests that no charge is collected within the dead layer, about half of the charge deposited in a dead layer diffuses into the active region (Wall et al., 2014). The non-ionizing radiation loss also causes surface defects that result in increased leakage current, which in turn causes reduced depletion of the detector and therefore incomplete charge collection (Coleman et al., 1968a, 1968b, Knoll, 2000; Wüest et al., 2007). The radiation damage to the detector thus results in a decrease in efficiency, in addition to the energy underestimate caused by the dead layer. Damage to the ohmic contact under high proton fluences can increase the contact resistance, another source of ohmic dissipation that decreases the depletion voltage (Coleman et al., 1968b).

While not offering any definitive explanations for the MAGPD degradation and the MAGED stability, this section quantifies the cumulative fluence observed by GOES-15
MAGPD, explores some aspects of the radiation damage, and reassesses older models in the light of these results.

\subsection{Comparison of MEPED and MAGPD exposures to $\mathrm{keV}$ proton fluences}

The GOES-15 MAGPD is the only MAGPD instrument for which observations are continuous since initial turn-on. Therefore, we use its data to estimate the cumulative fluences endured by the MAGPD telescope in geostationary orbit. Following Sandanger et al. (2015), we estimate the integral unidirectional flux in energy, in this case above $80 \mathrm{keV}$, by converting the MP1-MP5 differential fluxes from each telescope to integral fluxes within their bands, then summing them. Integral unidirectional daily fluence is calculated for each day, then accumulated and multiplied by the solid angle of each telescope $(0.112 \mathrm{sr})$ to produce the curves plotted in the left-hand panel of Figure 11. The changes in the slopes after 2-3 years are noticeable. This is a clear symptom of on-orbit instrument degradation since one would expect the slope if anything to increase heading into solar maximum, so the actual $>80 \mathrm{keV}$ fluence is much greater. The corrected fluences (calculated from the telescope fluxes after they are corrected using the intra-calibration scale factors and cross-calibrations with MPA, described above) are shown in the right-hand panel of Figure 11. After 8 years, the maximum corrected cumulative fluences are more than double the uncorrected values. The decrease in slope in 2018 is real and associated with low activity levels. The reordering of the curves results from the intra-calibrations, while the straightening out of the curves is due to the cross-calibrations with MPA. Since GOES-15 has undergone a yaw flip twice per year, one would expect the telescope pairs that swap orientation through each flip (2-4, $3-5,6-8$, and 7-9) to endure similar fluences, and this is apparent in the corrected fluences. Telescopes 7 and 9, which observed the lowest fluences, had pitch angles most nearly parallel or antiparallel to the magnetic field, in general. Telescopes 1, 2, 3, 4 and 5, which observed the greatest fluences, in general had pitch angles closer to $90^{\circ}$. This ordering of the fluence curves provides a further validation of the intra-calibration method.

From Figure 9 of Sandanger et al. (2015), a rough order-ofmagnitude estimate of the corrected monthly mean $>60 \mathrm{keV}$ flux is $10^{4} \mathrm{~cm}^{-2} \mathrm{~s}^{-1} \mathrm{sr}^{-1}$. This corresponds to an average daily fluence of $8.6 \times 10^{8} \mathrm{~cm}^{-2} \mathrm{sr}^{-1}$, which over 9 years accumulates to $\sim 2.8 \times 10^{12} \mathrm{~cm}^{-2} \mathrm{sr}^{-1}$ or $\sim 3.2 \times 10^{11} \mathrm{~cm}^{-2}$. All of the MAGPD telescopes endured this cumulative fluence within a few months of launch (Fig. 11). This illustrates the fact that a MAGPD telescope endures an order of magnitude greater proton fluence than a MEPED proton telescope over its lifetime. One reason for this difference is that MEPED telescopes pass through the proton aurora during a small fraction of every orbit, while MAGPD is almost continuously in the ring current, a source of the proton aurora (e.g., Kozyra et al., 1998). One consequence of this difference is that the assumption by Sandanger et al. (2015) of MEPED degradation being linear in cumulative fluence may not be valid for MAGPD over most of its lifetime.

\subsection{Proton damage versus depth in MAGPD and MAGED}

In order to gain some insight into the damage caused by the incident protons, we used the transport of ions in matter (TRIM) 

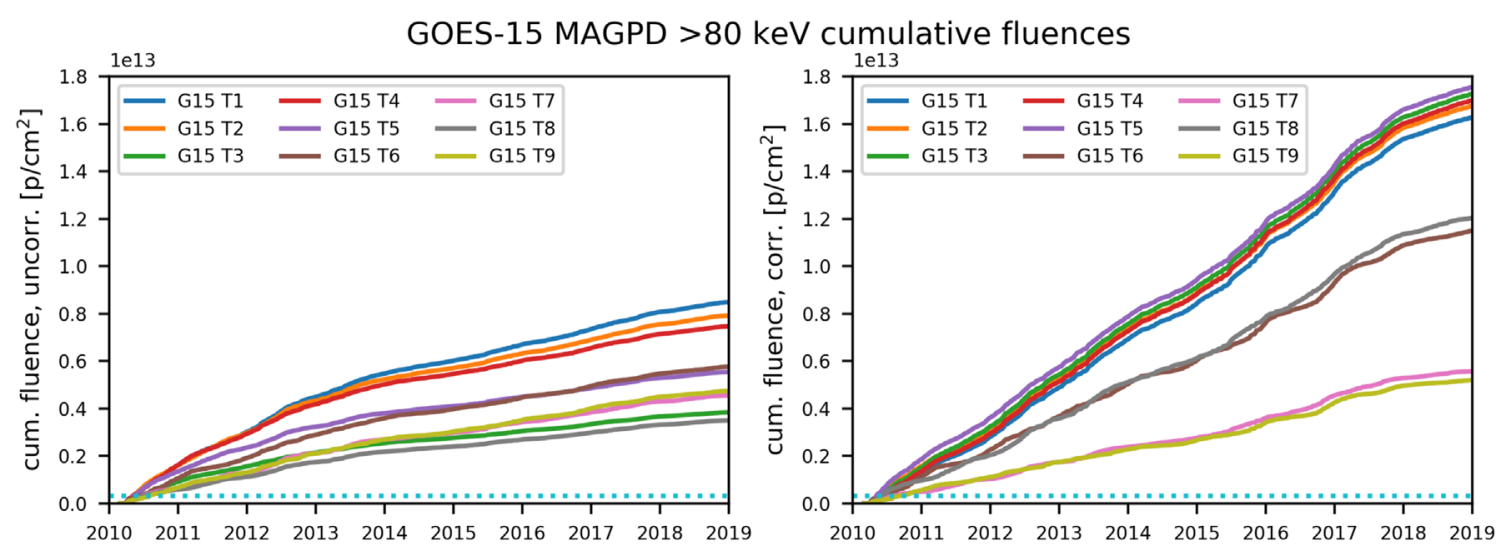

Fig. 11. Cumulative daily $>80 \mathrm{keV}$ proton fluences for each GOES-15 MAGPD telescope, March 2010 to December 2018, (left) uncorrected and (right) corrected. The dotted line indicates the approximate $>60 \mathrm{keV}$ proton fluence accumulated by a MEPED proton telescope in lowearth orbit over 9 years, estimated from Figure 9 of Sandanger et al. (2015).

Monte Carlo transport model (Ziegler et al., 2008) to calculate vacancies due to displacements as a function of depth in a MAGPD and MAGED telescope. One-dimensional calculations at $0^{\circ}$ incidence were used, with 10,000 runs for each curve. TRIM's "quick" calculation of target damage uses the modified Kinchin-Pease method (usually accurate to within a factor of 2) to calculate the number of vacancies per interval of depth per ion caused by ion-atom collisions and by collisions with the recoiling atom (Ziegler et al., 2008). The results are shown in Figure 12 for $250 \mathrm{keV}$ and lower energies over the first $3 \mu \mathrm{m}$ of total depth (Al $+\mathrm{Si}$ in MAGPD, $\mathrm{Ni}+\mathrm{Au}+\mathrm{Si}$ in MAGED). The protons are incident from the left. The bottom panel shows vacancies in MAGPD for $80-250 \mathrm{keV}$ protons. For all of the energies, the vast majority of the vacancies are in the Si detector. The protons in the MP1 channel (80-110 keV) cause most of their damage within the first $0.6 \mu \mathrm{m}$ of the $\mathrm{Si}$. For reference, most of the vacancies caused by $800 \mathrm{keV}$ protons lie between 10 and $12 \mu \mathrm{m}$ depth in the $\mathrm{Si}$ of the front detector (not shown).

The top panel shows vacancies in MAGED for $130-250 \mathrm{keV}$ protons. Very few of the $130 \mathrm{keV}$ protons reach the Si detector. At $187 \mathrm{keV}$, approximately half of the vacancies are in the $\mathrm{Ni}$ foil and half in the Si detector. At $250 \mathrm{keV}, 77 \%$ of the vacancies are in the $\mathrm{Si}$. Although $135 \mathrm{keV}$ has been cited as the lowest proton energy that penetrates the $\mathrm{Ni}$ foil (specifically, in the SEM-1 MEPED electron telescopes), $187 \mathrm{keV}$ is a more meaningful proton energy when considering damage to the MAGED Si detector. This is an important distinction since the $187 \mathrm{keV}$ proton fluxes are an order of magnitude lower than the $130 \mathrm{keV}$ fluxes. However, it is clear from the MAGPD observations that proton fluxes in this higher energy range are still substantially degraded. Two distinctions between MAGPD and MAGED may explain the apparent immunity of the latter to proton dose: (1) the surface damage that causes increased leakage current and reduced depletion may be caused by protons with energies $<187 \mathrm{keV}$ and (2) the much longer range of electrons in Si means that that most of the energy of incident electrons is deposited in volume mostly unaffected by proton dose.

\subsection{Radiation damage considerations}

Coleman et al. (1968a) characterized the effects of radiation damage by monoenergetic 50, 200,600, and $1000 \mathrm{keV}$ protons on the performance of surface barrier detectors with both the $\mathrm{Au}$ rectifying (surface barrier) contact and the $\mathrm{Al}$ ohmic contact facing the radiation source. The effects measured were current, noise, capacitance, and collection efficiency. With the Au contact facing the source, as the (monoenergetic) fluence increased above $10^{13} \mathrm{~cm}^{-2}$, detector noise, leakage current and capacitance increased substantially while collection efficiency decreased. The decreased collection efficiency was attributed to the increased leakage current resulting in degraded detector depletion. In the tests with the $\mathrm{Al}$ ohmic contact facing the source (at 200 and $800 \mathrm{keV}$ ), the fluence at which detector noise started to increase was three orders of magnitude greater than with the $\mathrm{Au}$ contact facing the source, and little degradation of depletion was observed. Coleman et al. (1968a) therefore recommended that surface barrier detectors be oriented with the $\mathrm{Al}$ ohmic contact facing space, while noting that the dead layer would prevent this arrangement from providing benefits at the proton energies causing the damage. At fluences above $10^{14} \mathrm{~cm}^{-2}$, the experimenters waited hours to days to characterize the effects until current and noise characteristics had stabilized (recovered partially) through annealing. Coleman et al. (1968b) extended the study to higher energies, starting at $800 \mathrm{keV}$, the upper end of the MAGPD energy range. Qualitatively, the results were similar, with small $(<1$ order-of-magnitude) changes in leakage current, noise and capacitance for $800 \mathrm{keV}$ fluences up to $10^{17} \mathrm{~cm}^{-2}$ when the Al ohmic contact was irradiated. These results supported the design recommendation of the first paper.

Despite following this recommendation, the MAGPD and MEPED proton telescopes (with the $\mathrm{Al}$ contact facing space) exhibited degradation at fluences $\left(10^{11}-10^{13} \mathrm{~cm}^{-2}\right)$ that were lower by more than three orders of magnitude than those fluences $\left(10^{16}-10^{17} \mathrm{~cm}^{-2}\right)$ observed by Coleman et al. (1968a) to cause measurable damage in the laboratory with the $\mathrm{Al}$ contact irradiated. This is a sobering result but does not negate the design recommendation. Comparisons between the laboratory and on-orbit results need to consider the observational differences. A major difference is the dose rate. The dose rate is much lower in space (the laboratory irradiations lasted $5 \mathrm{~min}$ ), and annealing (if it takes place) is a concurrent effect rather than observably separate as in the laboratory.

The five GOES-13 MAGPD channels exhibit a similar rate of degradation during the period of interest, as do the three 

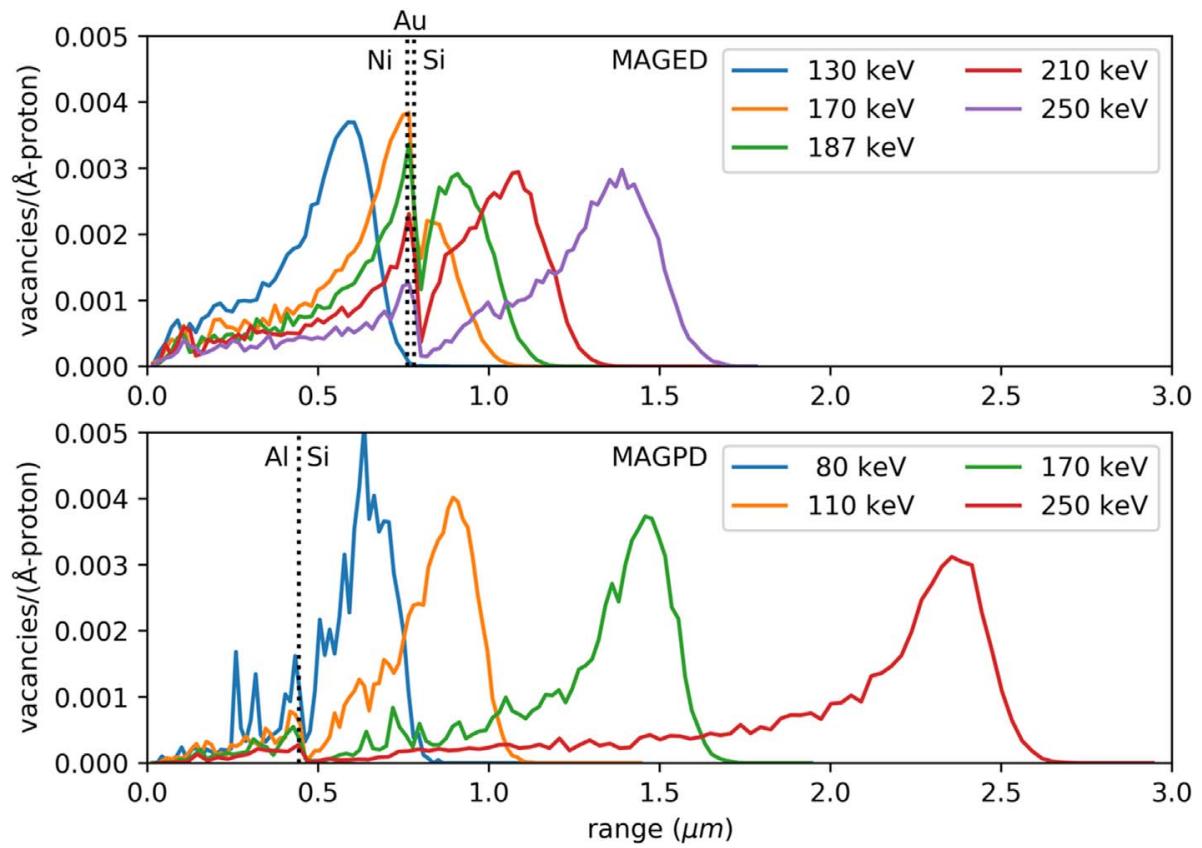

Fig. 12. Vacancies per unit depth per proton created by protons of selected proton energies in the front ends of the MAGED and MAGPD telescopes, simulated using the TRIM Monte Carlo model. For details, see the text.

lower-energy GOES-15 channels (Fig. 10). This runs counter to the model of a dead layer that has an energy-dependent effect on incident proton fluxes. The observed on-orbit degradation may be dominated at lower fluences by the dead layer and carrier trapping as postulated by the MEPED literature (Galand \& Evans, 2000; Wüest et al., 2007; Sandanger et al., 2015), while at greater fluences the less energy-dependent effects of leakage current and incomplete depletion may be more important. The observed partial recovery of the MAGPD channels after several years on orbit also contradicts a model of dead-layer degradation that is proportional to fluence. Therefore, the model of a completely dead layer reducing the energy deposited by protons, which has successfully guided the correction of MEPED proton fluxes (Sandanger et al., 2015), is inadequate to explain the observed degradation of MAGPD in the presence of orderof-magnitude greater proton fluences in GEO. A more complex model of the effects of radiation damage is needed to explain the MAGPD behavior, including partial charge collection from the dead layer, increased leakage current reducing in partial depletion of the active region, and partial recovery due perhaps to temperature-dependent annealing. Such a model would need to account both for decreases in detection efficiency and for effective energy shifts.

Future long-term proton observations should plan for correcting the on-orbit degradation of the observations. Proton solid-state telescopes should include features that permit diagnosis and correction for on-orbit degradation, such as reporting a subset of pulse heights for diagnosis and the ability to adjust detector bias. A weak alpha particle emitter illuminating the front detector would reveal (by its signatures in the pulse height data) energy degradation and an increase in the dead layer (Coleman et al., 1968a, 1968b). It may also be possible to derive better understanding of the effects of radiation damage from the physics of the fluxes themselves. For example, drift echoes observed commonly in the MAGPD fluxes could be used to determine the effective energies of the channels (O'Brien et al., 2015) as they change with time. This may permit the separation of the effects of efficiency degradation from energy shifts.

\section{Conclusions}

The GOES-13 and 15 MAGED (30-600 keV electrons) and MAGPD (80-800 keV protons) data sets were chosen to extend the energy range of an empirical geosynchronous plasma model upwards to include radiation belt energies. The existing model was derived from geosynchronous flux observations by the LANL MPA instrument (Denton et al., 2015, 2016). For incorporation into the model, the multi-telescope GOES fluxes were converted to omnidirectionally-averaged fluxes using the GOES magnetometer data. In order to ensure an accurate and internally consistent augmented empirical model, the following issues in the GOES data were identified and corrected for:

(a) Noise bursts that developed over time in two MAGED telescopes had to be removed. Data from one MAGED telescope had to be omitted entirely from the analysis. Sporadic periods of faulty magnetometer data also had to be omitted.

(b) Within each MAGED and MAGPD instrument, the nine telescopes had to be intra-calibrated by comparing observations when telescope pairs observe flux at the same pitch angle. The derived scale factors revealed annual as well as secular variations, the latter of which could have been due to on-orbit radiation damage. The multiplicative scale factors ranged from 0.48 to 1.8 for the electron telescopes and 0.19 to 2.8 for the proton telescopes. 
(c) GOES-13, -14 and -15 were cross-calibrated in July 2010 when they had been on-orbit 4 years, 1 year, and 4 months, respectively. Compared to the "fresh" GOES-15 observations, the GOES-13 and -14 electron fluxes exhibited no degradation. While the GOES-14 proton observations were also not degraded, the GOES-13 proton fluxes exhibited severe degradation, by a factor of 1.8-2.8, depending on energy, and were corrected for this prior to the next cross-calibration step.

(d) The GOES data were cross-calibrated with the LANL MPA data using a second-order fit to Bartels-rotation (27-day) flux averages. This fit was performed on 7 years of GOES-13 averages and 8 years of GOES-15 averages and on concurrent MPA data. Where they overlap in energy $(40 \mathrm{keV})$, the GOES electron fluxes agreed well with the LANL MPA electron fluxes over the entire period and required no correction. In contrast, the GOES proton fluxes exhibited significant degradation for up to 6 years on orbit, followed by a partial recovery. They were corrected by applying the ratios of the second-order fits in time.

The MAGPD telescopes endured an order-of-magnitude greater proton dose in GEO than the similar MEPED proton telescopes in low-earth orbit over a similar period. In combination with the on-orbit behavior of MAGPD and MAGED, this difference in dose suggests that the dead-layer model of radiation damage used successfully to correct MEPED proton data needs to be augmented with considerations of increased leakage current and reduced depletion of the surface-barrier detectors, and possibly on-orbit annealing, in order to understand the observed MAGPD degradation during 8+ years in GEO.

Acknowledgements. This material is based in part upon work supported by the National Aeronautics and Space Administration under Grant No. 80NSSC17K082 to the Space Science Institute (M. Denton, PI), under Subaward 00884 to the University of Colorado. The processing of the 2010 GOES fluxes was supported by the National Aeronautics and Space Administration under Grant No. NNX12AJ55G to UCLA (D. Turner, PI), under Subaward Agreement 2090 G QA024 to the University of Colorado. The GOES particle and magnetometer data used in this study are available from https:// ngdc.noaa.gov/stp/satellite/goes/dataaccess.html. JVR thanks W. Rowland for helpful discussions of the intra-calibration method. The editor thanks Bernard Blake and an anonymous reviewer for their assistance in evaluating this paper.

\section{References}

Bame SJ, McComas DJ, Thomsen MF, Barraclough BL, Elphic RC, Glore JP, Gosling JT, Chavez JC, Evans EP, Wymer FJ. 1993. Magnetospheric plasma analyzer for spacecraft with constrained resources. Rev Sci Instrum 64(4): 1026-1033. https://doi.org/ 10.1063/1.1144173.

Burin des Roziers E, Li X. 2006. Specification of $>2 \mathrm{MeV}$ geosynchronous electrons based on solar wind measurements. Space Weather 4: S06007. https://doi.og/10.1029/2005SW000177.
Coleman JA, Love DP, Trainor JH, Williams DJ. 1968a. Low-energy proton damage effects in silicon surface-barrier detectors. IEEE Trans Nucl Sci 15(1): 482-490. https://doi.org/10.1109/TNS. 1968.4324890.

Coleman JA, Love DP, Trainor JH, Williams DJ. 1968b. Effects of damage by $0.8 \mathrm{MeV}-5.0 \mathrm{MeV}$ protons in silicon surface barrier detectors. IEEE Trans Nucl Sci 15(3): 363-372. https://doi.org/ 10.1109/TNS.1968.4324960.

Denton MH, Thomsen MF, Jordanova VK, Henderson MG, Borovsky JE, Denton JS, Pitchford D, Hartley DP. 2015. An empirical model of electron and ion fluxes derived from observations at geosynchronous orbit. Space Weather 13: 233-249. https://doi.org/10.1002/2015SW001168.

Denton MH, Henderson MG, Jordanova VK, Thomsen MF, Borovsky JE, et al. 2016. An improved empirical model of electron and ion fluxes at geosynchronous orbit based on upstream solar wind conditions. Space Weather 14: 511-523. https://doi.org/ 10.1002/2016SW001409.

Denton MH, Taylor MGGT, Rodriguez JV, Henderson MG. 2019. Extension of an empirical electron flux model from 6 to 20 Earth radii using Cluster/RAPID observations. Space Weather 17: 778792. https://doi.org/10.1029/2018SW002121.

Evans DS, Greer MS. 2000. Polar Orbiting Environmental Satellite Space Environmental Monitor- 2: Instrument descriptions and archive data documentation, Tech. Mem., Natl. Atmos. and Oceanic Admin., Space Environ. Cent., Boulder, CO. https://satdat.ngdc. noaa.gov/sem/poes/docs/sem2_docs/2006/SEM2v2.0.pdf.

Galand M, Evans DS. 2000. Radiation damage of the proton MEPED detector on POES (TIROS/NOAA) satellites, NOAA Technical Report OAR 456-SEC 42, Boulder, CO.

Ganushkina NY, Sillanpää I, Welling DT, Haiducek J, Liemohn M, Dubyagin S, Rodriguez JV. 2019. Validation of inner magnetosphere particle transport and acceleration model (IMPTAM) with long-term GOES MAGED measurements of keV electron fluxes at geostationary orbit. Space Weather 17: 687-708. https://doi.org/ 10.1029/2018SW002028.

Ginet GP, O'Brien TP, Huston SL, Johnston WR, Guild TB, et al. 2013. AE9, AP9 and SPM: New models for specifying the trapped energetic particle and space plasma environment. Space Sci Rev 179: 579-615. https://doi.org/10.1007/s11214-013-9964-y.

Hanser FA. 2011. EPS/HEPAD calibration and data handbook, Tech. Rep. GOESN-ENG-048D, Assurance Technology Corporation, Carlisle, MA. Available from: https://ngdc.noaa.gov/stp/satellite/ goes/doc/goes_nop/GOESN-ENG-048_RevD_EPS_HEPAD_ 13May2011.pdf.

Hughes Aircraft Company. 1968. Applications technology satellite power subsystem radiation effects study. NASA Contract NAS 5-3823, SSD-80089R. Available from: https://ntrs.nasa.gov/.

Knoll GF. 2000. Radiation detection and measurement, 3rd edn., John Wiley \& Sons, Inc.

Korth H, Thomsen MF, Borovsky JE, McComas DJ. 1999. Plasma sheet access to geosynchronous orbit. J Geophys Res 104: 25047 25061. https://doi.org/10.1029/1999JA900292.

Kozyra JU, Fok MC, Sanchez ER, Evans DS, Hamilton DC, Nagy AF. 1998. The role of precipitation losses in producing the rapid early recovery phase of the Great Magnetic Storm of February 1986. J Geophys Res 103(A4): 6801-6814. https://doi.org/ 10.1029/97JA03330.

Liu DL, Liu SH, Panetta CJ, Hong SM, Olson KR, Alaan DR, Mann CJ, Luey KT. 2011. Synergistic effects of contamination and low energy space protons on solar cell current output. In: 37th IEEE Photovoltaic Specialists Conference, pp. 001595-001600. https://doi. org/10.1109/PVSC.2011.6186261. 
Lyons LR, Evans DS. 1984. An association between discrete aurora and energetic particle boundaries. J Geophys Res 89(A4): 23952400. https://doi.org/10.1029/JA089iA04p02395.

Matéo-Vélez J-C, Sicard A, Payan D, Ganushkina N, Meredith NP, Sillanpäa I. 2018. Spacecraft surface charging induced by severe environments at geosynchronous orbit. Space Weather 16: 89106. https://doi.org/10.1002/2017SW001689.

Meredith NP, Horne RB, Isles JD, Rodriguez JV. 2015. Extreme relativistic electron fluxes at geosynchronous orbit: Analysis of GOES E >2 MeV electrons. Space Weather 13: 170-184. https://doi.org/10.1002/2014SW001143.

NASA. 2011. Mitigating in-space charging effects - a guideline. NASA-HDBK-4002A. https://standards.nasa.gov/standard/nasa/nasahdbk-4002.

Nelder JA, Mead R. 1965. A simplex method for function minimization. Comput J 7(4): 308-313. https://doi.org/10.1093/comjnl/7. 4.308.

O'Brien TP, McPherron RL. 2003. An empirical dynamic equation for energetic electrons at geosynchronous orbit. J Geophys Res 108: 1137. https://doi.org/10.1029/2002JA009324.

O'Brien TP, Sornette D, McPherron RL. 2001. Statistical asynchronous regression: determining the relationship between two quantities that are not measured simultaneously. J Geophys Res 106: 13247-13259. https://doi.org/10.1029/2000JA900193.

O'Brien TP, Claudepierre SG, Looper MD, Blake JB, Fennell JF, et al. 2015. On the use of drift echoes to characterize on-orbit sensor discrepancies. J Geophys Res Space Phys 120: 2076-2087. https://doi.org/10.1002/2014JA020859.

O'Dell SL, Bautz MW, Blackwell WC Jr, Butt YM, Cameron RA, Elsner RF, Gussenhoven MS, Kolodziejczak JJ, Minow JI, Suggs RM, Swartz DA. 2000. Radiation environment of the Chandra X-ray Observatory. In: X-ray and gamma-ray instrumentation for astronomy XI, Vol. 4140, International Society for Optics and Photonics. pp. 99-110. https://doi.org/10.1117/12.409104.

Onsager TG, Chan AA, Fei Y, Elkington SR, Green JC, Singer HJ. 2004. The radial gradient of relativistic electrons at geosynchronous orbit. J Geophys Res 109: A05221. https://doi.org/ 10.1029/2003JA010368.

Panametrics. 2004a. Electron calibration report, GOES NO/PQ MAGED Telescope. GOESN-ENG-028. Available from: https:// ngdc.noaa.gov/stp/satellite/goes/documentation.html.

Panametrics. 2004b. Proton and electron calibration report, GOES NO/PQ MAGPD Telescope. GOESN-ENG-029. Available from: https://ngdc.noaa.gov/stp/satellite/goes/documentation.html.

Rodriguez JV. 2014. GOES 13-15 MAGE/PD pitch angles algorithm theoretical basis document, NOAA NESDIS National Geophysical Data Center. Available from: https://ngdc.noaa.gov/stp/satellite/ goes/documentation.html.

Rowland W, Weigel RS. 2012. Intracalibration of particle detectors on a three-axis stabilized geostationary platform. Space Weather 10(11): S11002. https://doi.org/10.1029/2012SW000816.
Sandanger MI, Ødegaard LKG, Nesse Tyssøy H, Stadsnes J, Søraas F, Oksavik K, Aarsnes K. 2015. In-flight calibration of NOAA POES proton detectors-Derivation of the MEPED correction factors. J Geophys Res Space Phys 120: 9578-9593. https://doi. org/10.1002/2015JA021388.

Seale RA, Bushnell RH. 1987. The TIROS-N / NOAA A-J space environment monitor subsystem, NOAA Technical Memorandum ERL SEL-75. Available from: https://ngdc.noaa.gov/stp/satellite/ poes/documentation.html.

Sen PK. 1968. Estimates of the Regression Coefficient Based on Kendall's Tau. J Am Stat Assoc 63: 1379-1389. https://doi.org/ 10.1080/01621459.1968.10480934.

Sibeck DG, McEntire RW, Lui ATY, Lopez RE, Krimigis SM. 1987. Magnetic field drift shell splitting: Cause of unusual dayside particle pitch angle distributions during storms and substorms. $J$ Geophys Res Space Phys 92: 13485-13497. https://doi.org/ 10.1029/JA092iA12p13485.

Sillanpää I, Ganushkina NY, Dubyagin S, Rodriguez JV. 2017. Electron fluxes at geostationary orbit from GOES MAGED data. Space Weather 15: 1602-1614. https://doi.org/10.1002/2017SW001698.

Statler RL, Curtin DJ. 1971. Radiation damage in silicon solar cells from low-energy protons. IEEE Trans. Elect Dev ED 18: 412-417. https://doi.org/10.1109/T-ED.1971.17217.

Tada HY, Carter JR Jr, Anspaugh BE, Downing RG. 1982. Solar cell radiation handbook, 3rd edn., JPL Publication 82-69. Available from: https://ntrs.nasa.gov/.

Thomsen MF, Noveroske E, Borovsky JE, McComas DJ. 1999. Calculation of moments from measurements by the Los Alamos magnetospheric plasma analyzer, Los Alamos National Lab. Rept. LA-13566-MS, Los Alamos, NM. https:/www.osti.gov/servlets/ purl/8188.

Wall BL, Amsbaugh JF, Beglarian A, Bergmann T, Bichsel HC, et al. 2014. Dead layer on silicon $\mathrm{p}-\mathrm{i}-\mathrm{n}$ diode charged-particle detectors. Nucl Instrum Meth Phys Res Sect A Accel Spectr Detect Assoc Equip 744: 73-79. https://doi.org/10.1016/j.nima.2013.12.048.

Wüest M, Evans DS, von Steiger R (Eds.). 2007. Calibration of particle instruments in space physics, ISSI Scientific Report, SR007. http://www.issibern.ch/PDF-Files/SR-007.pdf.

Zhao H, Li X, Baker DN, Fennell JF, Blake JB, et al. 2015. The evolution of ring current ion energy density and energy content during geomagnetic storms based on Van Allen Probes measurements. J Geophys Res Space Phys 120: 7493-7511. https://doi.org/ 10.1002/2015JA021533.

Zheng Y, Ganushkina NY, Jiggens P, Jun I, Meier M, et al. 2019. Space radiation and plasma effects on satellites and aviation: Quantities and metrics for tracking performance of space weather environment models. Space Weather 17: 1384-1403. https://doi. org/10.1029/2018SW002042.

Ziegler JF, Biersack JP, Ziegler MD. 2008. SRIM: The stopping and range of ions in matter, SRIM Co. www.SRIM.org.

Cite this article as: Rodriguez JV, Denton MH \& Henderson MG 2020. On-orbit calibration of geostationary electron and proton flux observations for augmentation of an existing empirical radiation model. J. Space Weather Space Clim. 10, 28. 